THE ASTROPHYSICAL JOURNAL, 546:469-483, 2001 January 1

(c) 2001. The American Astronomical Society. All rights reserved. Printed in U.S.A.

\title{
GRAVITY MODES IN ZZ CETI STARS. IV. AMPLITUDE SATURATION BY PARAMETRIC INSTABILITY
}

\author{
YANQIN Wu ${ }^{1,2}$ AND Peter GoldReich ${ }^{3}$ \\ Received 2000 March 12; accepted 2000 August 10
}

\begin{abstract}
ZZ Ceti stars (also known as DAV stars) exhibit small-amplitude photometric pulsations in multiple gravity modes. As the stars cool, their dominant modes shift to longer periods. We demonstrate that parametric instability limits overstable modes to amplitudes similar to those observed. In particular, it reproduces the trend that longer period modes have larger amplitudes. Parametric instability is a form of resonant three-mode coupling. It involves the destabilization of a pair of stable daughter modes by an overstable parent mode. The three modes must satisfy exact angular selection rules and approximate frequency resonance. The lowest instability threshold for each parent mode is provided by the daughter pair that minimizes $\left(\delta \omega^{2}+\gamma_{d}^{2}\right) / \kappa^{2}$, where $\kappa$ is the nonlinear coupling constant, $\delta \omega$ is the frequency mismatch, and $\gamma_{d}$ is the energy damping rate of the daughter modes. Parametric instability leads to a steady state if $|\delta \omega|>\gamma_{d}$ and to limit cycles if $|\delta \omega|<\gamma_{d}$. The former behavior characterizes low radial order $(n \leq 3)$ parent modes, and the latter those with $n \geq 5$. In either case, the overstable mode's amplitude is maintained at close to the instability threshold value. Although parametric instability defines an upper envelope for the amplitudes of overstable modes in ZZ Ceti stars, other nonlinear mechanisms are required to account for the irregular distribution of amplitudes of similar modes and the nondetection of modes with periods longer than $1200 \mathrm{~s}$. Resonant three-mode interactions involving more than one excited mode may account for the former and Kelvin-Helmholtz instability of the mode-driven shear layer below the convection zone for the latter.
\end{abstract}

Subject headings: instabilities - stars: oscillations - white dwarfs

\section{INTRODUCTION}

Within an instability strip of width $\Delta T_{\text {eff }} \approx 10^{3} \mathrm{~K}$ centered at $T_{\text {eff }} \approx 1.2 \times 10^{3} \mathrm{~K}$, hydrogen white dwarfs exhibit multiple excited gravity modes with $10^{2} \lesssim P \lesssim 10^{3} \mathrm{~s}$. Convective driving, originally proposed by Brickhill (1990, 1991), is the overstability mechanism (Goldreich \& Wu 1998, hereafter Paper I). Individual modes maintain small amplitudes; typical fractional flux variations range from a few millimodulation amplitudes to a few tens of millimodulation amplitudes. ${ }^{4}$

The nonlinear mechanism responsible for saturating mode amplitudes has not previously been identified. We demonstrate that parametric resonance between an overstable parent $g$-mode and a pair of lower frequency damped daughter $g$-modes sets an upper envelope to the parent modes' amplitudes. ${ }^{5}$ Moreover, the envelope we calculate reproduces the broad trends found from observational determinations of mode amplitudes in ZZ Ceti stars. Our investigation follows pioneering work by Dziembowski \& Krolikowska (1985) on overstable acoustic modes in $\delta$ Scuti stars. They showed that parametric resonance with damped daughter $g$-modes saturates the growth of the overstable $p$-modes at approximately their observed amplitudes.

This paper is comprised of the following parts. In $\S 2$ we introduce parametric instability for a pair of damped

\footnotetext{
${ }^{1}$ Astronomy Unit, School of Mathematical Sciences, Queen Mary and Westfield College, London, UK.

${ }^{2}$ Canadian Institute of Theoretical Astrophysics, University of Toronto, 60 St. George Street, Toronto, Ontario M5S 3H8, Canada; wu@cita.utoronto.ca.

${ }^{3}$ Theoretical Astrophysics, California Institute of Technology 130-33, Pasadena, CA 91125; pmg@gps.caltech.edu.

${ }^{4}$ One millimodulation amplitude ( $\mathrm{mma}$ ) equals $0.1 \%$ fractional change in flux (Winget et al. 1994).

${ }^{5}$ The $g$-mode dispersion relation allows plenty of good resonances.
}

daughter modes resonantly coupled to an overstable parent mode. We evaluate the parent mode's threshold amplitude and describe the evolution of the instability to finite amplitude. Section 3 is devoted to the choice of optimal daughter pairs. We discuss relevant properties of three-mode coupling coefficients and the constraints imposed by frequency resonance relations and angular selection rules. Evaluation of the upper envelope for parent mode amplitudes set by parametric resonance is the subject of $\S 4$. Numerical results are interpreted in terms of analytic scaling relations and compared to observations. Section 5 contains a discussion of a variety of issues left over from this investigation. Detailed derivations are relegated to a series of appendices.

The stellar models used in this investigation were provided by Bradley (1996). Their essential characteristics are $M_{*}=0.6 M_{\odot}, \log \left(\mathrm{g} / \mathrm{cm} \mathrm{s}^{-2}\right)=8.0$, hydrogen layer mass $1.5^{*} \times 10^{-4} M_{*}$, and helium layer mass $1.5 \times 10^{-2} M_{*}$.

\section{PARAMETRIC INSTABILITY}

In this section we introduce parametric instability (Landau \& Lifshitz 1976). We present the threshold criterion for the instability and discuss relevant aspects of the subsequent evolution. Depending upon the parameters, the modes either approach a stable steady state or develop limit cycles. We describe the energies attained by the parent and daughter modes in either case. Many of the results in this section were obtained earlier by Dziembowski (1982).

\subsection{Instability Threshold}

Parametric instability in the context of our investigation is a special form of resonant three-mode coupling. It refers to the destabilization of a pair of damped daughter modes by an overstable parent mode. The frequencies of the three modes satisfy the approximate resonance condition $\omega_{p} \approx$ $\omega_{d_{1}}+\omega_{d_{2}}$, where the subscripts $p, d_{1}$, and $d_{2}$ denote one parent and two daughter modes, respectively. 


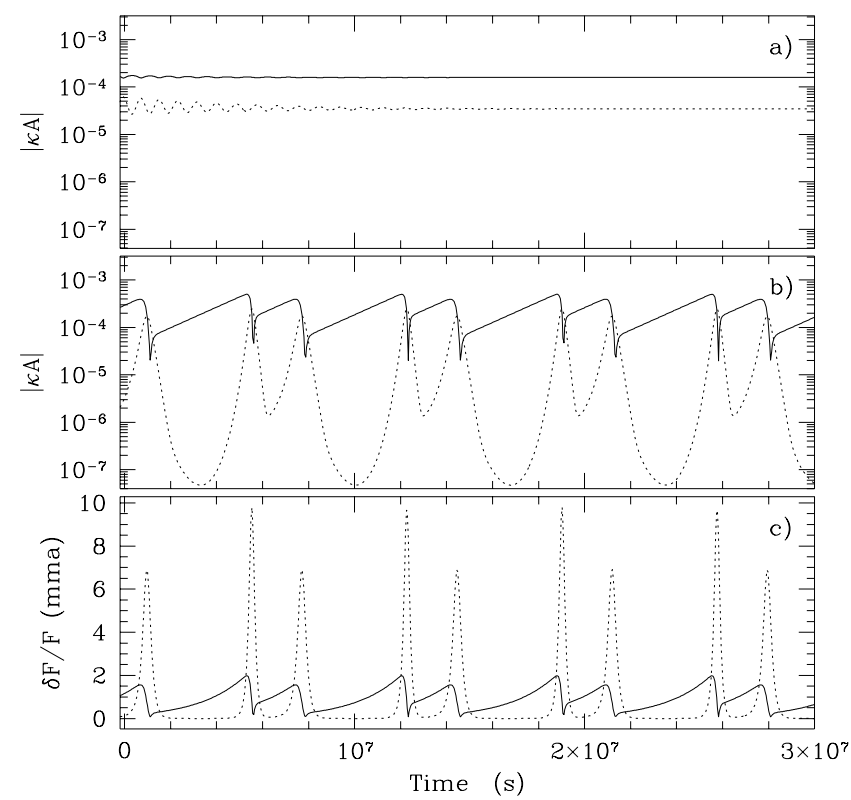

FIG. 1.-Parametric instability studied by numerical integration of eqs. (1)-(3). Solid lines represent parent mode amplitudes and dashed lines those of daughter modes. We multiply these amplitudes with $\kappa$ to make them dimensionless. For the top panel $\delta \omega=\omega_{d_{1}}+\omega_{d_{2}}-\omega_{p}=2 \times 10^{-5}$ $\mathrm{s}^{-1} \gg \gamma_{d_{1}}=\gamma_{d_{2}}=10^{-6} \mathrm{~s}^{-1}$, and the system settles into a steady state. The middle panel shows a case with $\delta \omega=10^{-6} \mathrm{~s}^{-1} \ll \gamma_{d_{1}}=\gamma_{d_{2}}=10^{-5} \mathrm{~s}^{-1}$, for which the mode amplitudes undergo limit cycles. These panels illustrate the two types of behavior discussed in $\S 2.2$. The bottom panel displays photospheric flux variations associated with the case shown in the middle panel. The daughter mode energies episodically approach that of the parent mode. Around these times their fractional flux amplitudes exceed that of the parent mode because they have smaller mode masses. For both simulations, we take mode periods to be $P_{p}=500 \mathrm{~s}^{-1}, P_{d_{2}} / P_{d_{1}}=0.9$, and $\gamma_{p}=10^{-7} \mathrm{~s}^{-1}$.

Equations governing the temporal evolution of mode amplitudes are most conveniently derived from an action principle (Newcomb 1962; Kumar \& Goldreich 1989). The amplitude equations take the form

$$
\begin{aligned}
& \frac{d A_{p}}{d t}=+\frac{\gamma_{p}}{2} A_{p}-i \omega_{p} A_{p}+i \frac{3}{\sqrt{2}} \omega_{p} \kappa A_{d_{1}} A_{d_{2}}, \\
& \frac{d A_{d_{1}}}{d t}=-\frac{\gamma_{d_{1}}}{2} A_{d_{1}}-i \omega_{d_{1}} A_{d_{1}}+i \frac{3}{\sqrt{2}} \omega_{d_{1}} \kappa A_{p} A_{d_{2}}^{*}, \\
& \frac{d A_{d_{2}}}{d t}=-\frac{\gamma_{d_{2}}}{2} A_{d_{2}}-i \omega_{d_{2}} A_{d_{2}}+i \frac{3}{\sqrt{2}} \omega_{d_{2}} \kappa A_{p} A_{d_{1}}^{*} .
\end{aligned}
$$

Here $A_{j}$ is the complex amplitude of mode $j$; it is related to the mode energy $E_{j}$ by $\left|A_{j}\right|^{2}=E_{j}$. The $\gamma_{j}(>0)$ denote linear energy growth and damping rates, and $\kappa$ is the nonlinear coupling constant (see $\S 3.1$ ). Our amplitude equations differ only in notation from those given by Dziembowski (1982).

The instability threshold follows from a straightforward linear stability analysis applied to equations (2) and (3). The effects of nonlinear interactions on the parent mode are ignored as is appropriate for infinitesimal daughter mode amplitudes. The critical parent mode amplitude satisfies (Vandakurov 1979; Dziembowski 1982)

$$
\left|A_{p}\right|^{2}=\frac{\gamma_{d_{1}} \gamma_{d_{2}}}{18 \kappa^{2} \omega_{d_{1}} \omega_{d_{2}}}\left[1+\left(\frac{2 \delta \omega}{\gamma_{d_{1}}+\gamma_{d_{2}}}\right)^{2}\right]
$$
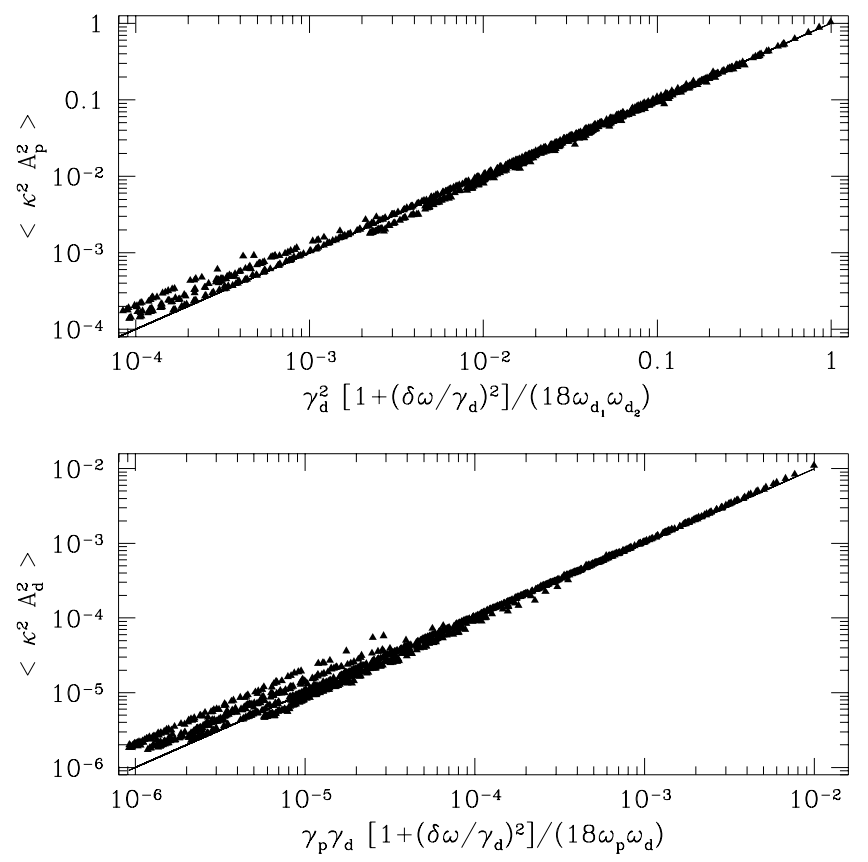

FIG. 2.-Numerical simulations confirm theoretical predictions for time-averaged energies of modes undergoing parametric instability. We numerically integrate eqs. (1)-(3) for a variety of three-mode systems with different frequencies and energy growth/decay rates. Each point on the figure represents one such system. We restrict ourselves to systems for which $\gamma_{d_{1}}=\gamma_{d_{2}}=\gamma_{d}$ because these are most relevant to our application (see $\S 3.2$ ). Time-averaged energies of parent and daughter modes are plotted against theoretically predicted values (solid lines; eqs. [5]-[7]) in the upper and lower panels, respectively. Energies multiplied by $\kappa^{2}$ are dimensionless. The subscript " $d$ " in the lower panel is taken to be $d_{1}$ or $d_{2}$ as in eqs. (6) and (7).

where $\delta \omega \equiv \omega_{d_{1}}+\omega_{d_{2}}-\omega_{p}$. The slower the daughter modes damp, the easier they are to excite.

\subsection{Dynamics}

The amplitude equations (1)-(3) have a unique equilibrium solution given by

$$
\begin{gathered}
\left|A_{p}\right|^{2}=\frac{\gamma_{d_{1}} \gamma_{d_{2}}}{18 \kappa^{2} \omega_{d_{1}} \omega_{d_{2}}}\left[1+\left(\frac{2 \delta \omega}{\gamma_{d_{1}}+\gamma_{d_{2}}-\gamma_{p}}\right)^{2}\right], \\
\left|A_{d_{1}}\right|^{2}=\frac{\gamma_{d_{2}} \gamma_{p}}{18 \kappa^{2} \omega_{d_{2}} \omega_{p}}\left[1+\left(\frac{2 \delta \omega}{\gamma_{d_{1}}+\gamma_{d_{2}}-\gamma_{p}}\right)^{2}\right], \\
\left|A_{d_{2}}\right|^{2}=\frac{\gamma_{d_{1}} \gamma_{p}}{18 \kappa^{2} \omega_{d_{1}} \omega_{p}}\left[1+\left(\frac{2 \delta \omega}{\gamma_{d_{1}}+\gamma_{d_{2}}-\gamma_{p}}\right)^{2}\right],
\end{gathered}
$$

together with

$$
\cot \Phi=-\frac{2 \delta \omega}{\gamma_{d_{1}}+\gamma_{d_{2}}-\gamma_{p}} .
$$

Here $\Phi=\theta_{d_{1}}+\theta_{d_{2}}-\theta_{p}$, where the complex amplitude $A_{j}$ may be written as $A_{j}=\left|A_{j}\right| e^{-i \theta_{j}}$. Note that for $\gamma_{p} \ll \gamma_{d}$ (which is the limit that concerns us; see below), equation (5) is identical to the threshold criterion (eq. [4]). Here $\gamma_{d}=\left(\gamma_{d_{1}}\right.$ $\left.+\gamma_{d_{2}}\right) / 2$ is the characteristic damping rate for the daughter modes. It is worth noting that, in this limit, the parent mode energy is independent of $\gamma_{p}$.

Provided $\gamma_{p} \ll \gamma_{d}$, the equilibrium state is a stable attractor for mode triplets with $|\delta \omega|>\gamma_{d}$, and unstable otherwise (Wersinger, Finn, \& Ott 1980; Dziembowski 1982). 
Figures $1 a$ and $1 b$ illustrate these two types of behaviors. Triplets with unstable equilibria undergo a variety of limit cycles. These share a number of common features. The parent mode's amplitude remains close to its equilibrium (threshold) value, with slow rises on timescale $\gamma_{p}^{-1}$ followed by precipitous drops on timescale $\gamma_{d}^{-1}$. The daughter modes' amplitudes stay far below their equilibrium values for most of the cycle but peak with amplitudes comparable to that of the parent mode for a brief interval of length $\gamma_{d}^{-1}$ shortly after the parent mode amplitude reaches its maximum value. During this brief interval, the energy that the parent mode has slowly accumulated is transferred to and dissipated by the daughter modes. So we see that, independent of its stability, the equilibrium state defines the parent mode's amplitude. This is confirmed by Figure $2 a$. We employ this result in $\S 3$ where we predict upper limits for $g$-mode amplitudes in pulsating white dwarfs. The timeaveraged energies of the daughter modes are also found to be consistent with equations (6) and (7) (Fig. 2b).

For $\gamma_{p} \geq 2 \gamma_{d}$, the equilibrium solution (eqs. [5]-[7]) indicates $E_{d} \geq E_{p}$. As nonlinear mode couplings promote energy equipartition, this would imply that, on average, the stable daughter modes transfer energy to the overstable parent mode. Numerical simulation indicates that in this limit, parametric instability does not lead to saturation of the parent mode amplitude. Our empirical finding is that saturation generally occurs provided $\gamma_{p} \leq 0.25 \gamma_{d}$. We adopt this criterion in applications made later in this paper.

If the daughter modes suffer nonlinear dissipation in addition to linear damping, both the dynamics and the equilibrium amplitudes will be modified. We discuss this complication in $\S 5$.

\section{CHOOSING THE BEST DAUGHTER PAIRS}

The discussion in the previous section shows that an overstable parent mode's amplitude saturates at a value close to the threshold for parametric instability. Although each overstable parent mode has many potential daughter pairs, the most important pair is the one with the lowest instability threshold. This section is devoted to identifying these optimal daughter pairs, a task that separates into two independent parts, maximization of $\kappa^{2}$ and minimization of $\left(\delta \omega^{2}+\gamma_{d}^{2}\right)$.

\subsection{Three-Mode Coupling Coefficients}

The three-mode coupling coefficient characterizes the lowest order nonlinear interactions among stellar modes. A compact form suitable for adiabatic modes under the Cowling approximation is derived in Kumar \& Goldreich (1989):

$$
\begin{aligned}
\kappa= & -\int d^{3} x \frac{p}{6}\left[\left(\Gamma_{1}-1\right)^{2}(\nabla \cdot \xi)^{3}\right. \\
& \left.+3\left(\Gamma_{1}-1\right)(\nabla \cdot \xi) \xi_{; j}^{i} \xi_{; i}^{j}+2 \xi_{; j}^{i} \xi_{; k}^{j} \xi_{; i}^{k}\right],
\end{aligned}
$$

where $p$ is the unperturbed pressure, $\Gamma_{1}$ is the adiabatic index, $\xi$ is the Lagrangian displacement, the subscript semicolon denotes covariant derivative, and the integration is over the volume of the star. This expression for $\kappa$ is symmetric with respect to the three modes. Note that the displacements enter only through components of their gradients. The present form is not suitable for accurate numerical computation. We derive a more appropriate version in $\S A 1$ (eq. [A15]).
Each eigenmode of a spherical star is characterized by three eigenvalues $n, \ell$, and $m ; n$ is the number of radial nodes in the radial displacement eigenfunction, $\ell$ is the spherical degree, and $m$ is the azimuthal number with the angular dependence described by a spherical harmonic $Y_{\ell m}(\theta, \phi)$. Integration over solid angle enforces the following selection rules on triplets with nonvanishing $\kappa: \mid \ell_{d_{2}}$ $-\ell_{d_{1}} \mid \leq \ell_{p} \leq \ell_{d_{1}}+\ell_{d_{2}}$ with $\ell_{p}+\ell_{d_{1}}+\ell_{d_{2}}$ even and $m_{p}=$ $m_{d_{1}}+m_{d_{2}}$ (see $\left.\S \mathrm{A} 1\right)$. These selection rules guarantee the conservation of angular momentum during nonlinear interactions. The magnitude of $\kappa$ is largest when the eigenfunctions of the daughter modes are radially similar in the upper evanescent zone of the parent mode. Radial similarity requires near equality of the vertical components of WKB wavevectors, $k_{z}$, where for gravity modes $k_{z}^{2} \approx\left(N^{2} / \omega^{2}\right.$ $-1) \Lambda^{2} / r^{2}$ (eq. [A2] of Paper I) with $N^{2}$ being the BruntVäisälä frequency, $r$ the radius, and $\Lambda^{2}=\ell(\ell+1)$. We take $N \sim \omega_{p}$ as the major contribution to the peak value of $|\kappa|$ comes from the region just above $z_{\omega_{p}}$, the upper boundary of the parent mode's propagating cavity. The $g$-mode dispersion relation applicable for $\omega \ll 10^{-2} \mathrm{~s}^{-1}$ is $\omega \propto \ell / n$ (see Fig. 4 of Paper I). We find that $\kappa$ is largest when

$$
\frac{n_{d_{1}}}{n_{d_{2}}} \sim \frac{\Lambda_{d_{1}} \omega_{d_{1}}}{\Lambda_{d_{2}} \omega_{d_{2}}} \sim\left(\frac{\omega_{p}^{2}-\omega_{d_{1}}^{2}}{\omega_{p}^{2}-\omega_{d_{2}}^{2}}\right)^{1 / 2} .
$$

Taking $\ell_{p}=1, \ell_{d_{2}}=\ell_{d_{1}}+1$, and evaluating the above relation for $\ell_{d_{1}}<10$, we locate the peak of $\kappa$ to be at

$$
n_{d_{1}}-n_{d_{2}} \approx-0.7 n_{p} \text {. }
$$

The width of this peak when measured in $n_{d_{1}}-n_{d_{2}}$ is of order $n_{p}$ because a fraction $n_{p}^{-1}$ of the nodes of each daughter mode lie in the region above $z_{\omega_{p}}$ (see discussion in $\S \mathrm{A} 2$ ). Figure 3 illustrates the behavior of $\kappa$ for a variety of combinations of parent and daughter modes.

As we show in $\S A 2$, the maximum value of $|\kappa|$ is of order

$$
|\kappa|_{\max } \sim \frac{1}{\left(n_{p}^{3} L \tau_{\omega_{p}}\right)^{1 / 2}} .
$$

Here $L$ is the stellar luminosity and $\tau_{\omega_{p}}$ is the thermal timescale at $z_{\omega_{p}}$. This analytical estimate is compared with numerical results in Figure 4. Note that $|\kappa|_{\max }$ depends entirely upon the properties of the parent mode and not at all upon those of its daughters. Our analytic estimate for $|\kappa|_{\max }$ assumes that mode trapping is negligible. Its good fit to the numerical results validates this assumption.

\subsection{Frequency Mismatch and Damping Rates}

Because the maximum value of $|\kappa|$ is independent of $\ell_{d_{1}}$ and $\ell_{d_{2}}$, we choose these parameters to minimize $\left(\delta \omega^{2}+\gamma_{d}^{2}\right)$. Radial similarity of the daughter modes to which a given parent mode couples most strongly (eq. [10]) implies $\gamma_{d_{1}} \approx$ $\gamma_{d_{2}}$.

Consider an $\ell_{p}, m_{p}$ parent mode. For each choice of $\ell_{d_{1}}, \ell_{d_{2}}$, there are of order $\ell_{d_{1}} n_{p}^{2}$ daughter pairs for which $\kappa$ is close to its maximum value. The factor $\ell_{d_{1}}$ arises from the freedom in choosing $m_{d_{1}}$, while the factor $n_{p}^{2}$ comes from the width of maximum $|\kappa|$ at each $\ell_{d_{1}}$. Relaxing the value of $\ell_{d_{2}}$ subject to the constraint of the angular selection rules increases the number of pairs by a factor of order $\ell_{p}$. Now replace $\ell_{d_{1}}$ by a running variable $\ell_{d_{1}}^{\prime}$. The number of pairs with $\ell_{d_{1}}^{\prime} \leq \ell_{d_{1}}$ is of order $\ell_{p} \ell_{d_{1}}^{2} n_{p}^{2}$. The distribution of the $\delta \omega$ values of these pairs is uniform between 0 and 

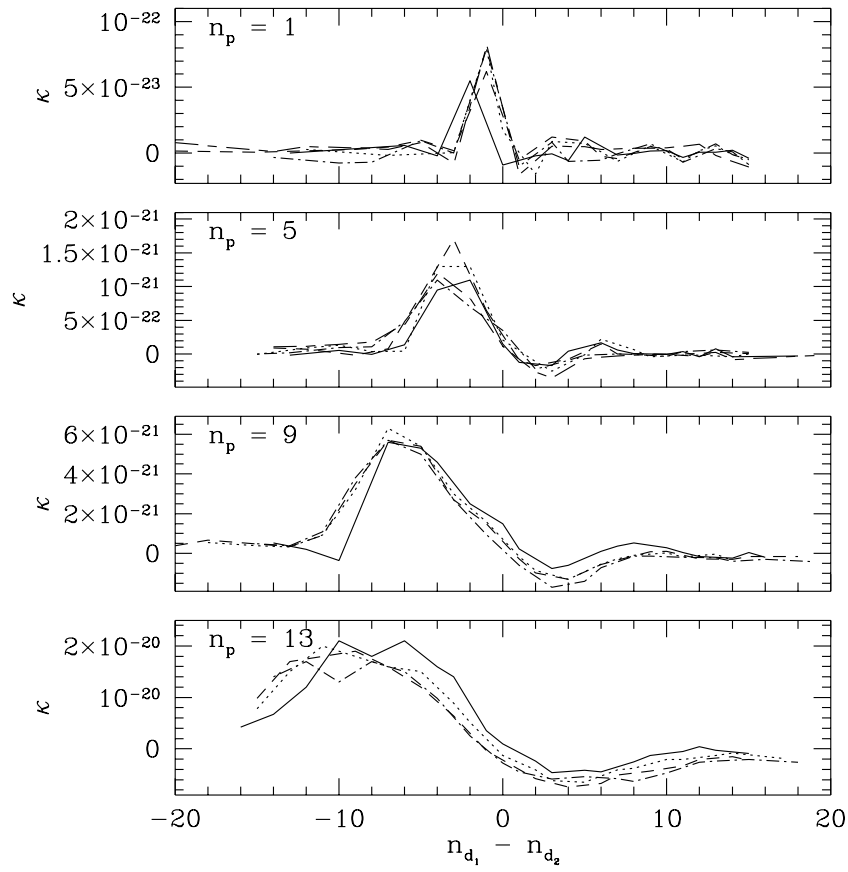

FIG. 3.-Coupling coefficient $\kappa$ expressed in ergs $^{-1 / 2}$ calculated from eq. (A15) for a DA white dwarf model with $T_{\text {eff }}=12,800 \mathrm{~K}$. From top to bottom panels, we consider four $\ell_{p}=1$ parent modes with increasing $n_{p}$. Coupling coefficients between each parent and its daughter pairs are plotted vs. $n_{d_{1}}-n_{d_{2}}$. Solid lines denote daughter pairs with $\ell_{d_{1}}=1$, and others those with $\ell_{d_{1}}=2,3,4,5$, and 9. Mode 3 is chosen to minimize $|\delta \omega|$ subject to the constraint $\ell_{d_{2}}=\ell_{p}+\ell_{d_{1}} ; \kappa$ is seen to depend on the radial similarity of the two daughter modes (eq. [10]) but not on their spherical degrees. The FWHM of the peak in $|\kappa|$ is of order $n_{p}$. Maximum $|\kappa|$ occurs for $n_{d_{1}}-n_{d_{2}} \sim-0.7 n_{p}$ (eq. [11]).

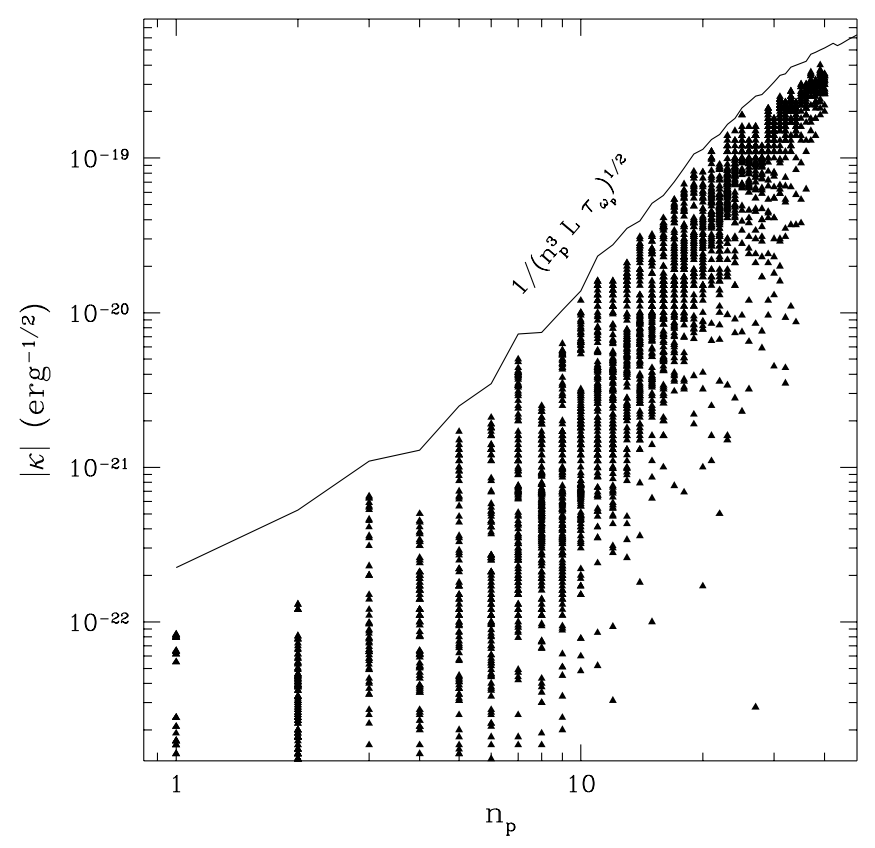

FIG. 4.-Coupling coefficients as a function of parent mode's radial order. We consider $\ell_{p}=1$ parent modes and include all daughter pairs that have $\ell_{d_{1}}=1, \ldots, 9$ and $\ell_{d_{2}}=\ell_{p}+\ell_{d_{1}}$. For each mode 2 , mode 3 is chosen to minimize $|\delta \omega|$. The solid line corresponds to the theoretical estimate for the maximum $|\kappa|$ at each $n_{p}$ (eq. [12]). These calculations are based on the same stellar model used for Fig. 3.
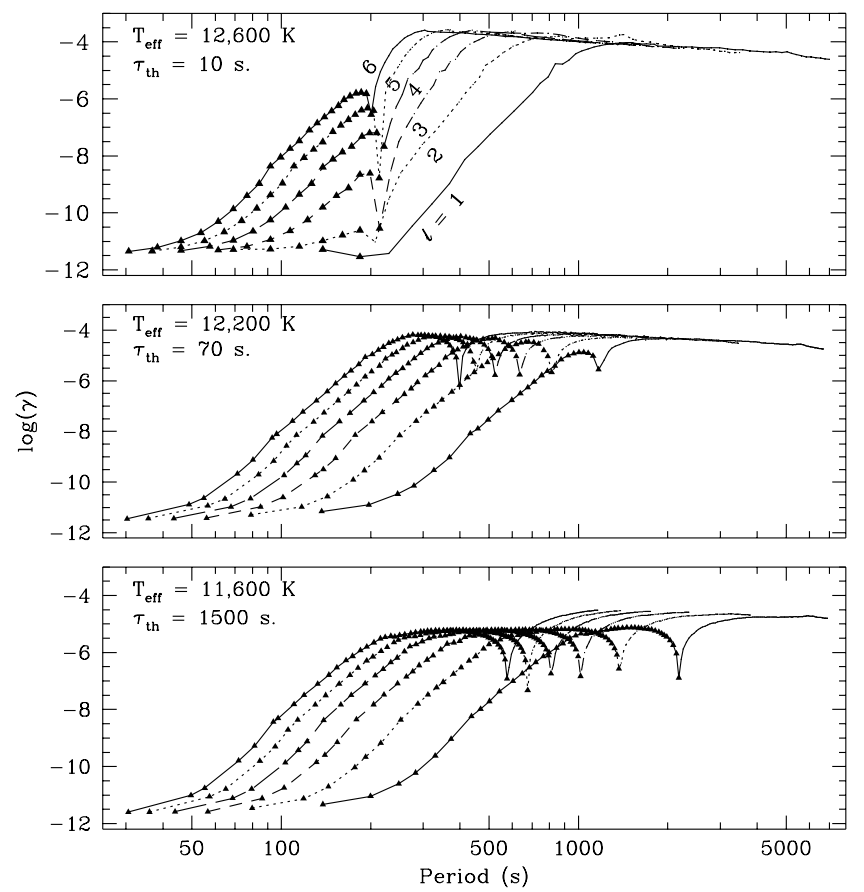

FIG. 5.-Nonadiabatic energy driving and damping rates as a function of mode period. See Paper II for computational details. We consider three stellar models with decreasing effective temperature; $\tau_{\text {th }}$ is the thermal timescale at the bottom of the convective zone. Overstable modes are marked by filled triangles. Lines connect points associated with modes of the same $\ell$. For each $\ell, \gamma$ exhibits an initial steep rise with increasing mode period (eq. [14]), followed by a gradual decline (eq. [15]). Maximum $\gamma$ occurs at the transition between quasi-adiabatic and strongly nonadiabatic damping. The latter regime is discussed in $\S \mathrm{B} 1$.

$n_{p} \omega_{d_{1}} / n_{d_{1}} \cdot{ }^{6}$ Statistically, the minimum frequency mismatch

$$
\Delta \omega \sim \frac{\omega_{d_{1}}}{n_{d_{1}}} \frac{1}{\ell_{p} \ell_{d_{1}}^{2} n_{p}} \sim \frac{\omega_{p}}{\ell_{d_{1}}^{3} n_{p}^{2}},
$$

where the low-frequency limit of the dispersion relation, $\omega \propto \ell / n$, is assumed in going from the first to the second relation for $\Delta \omega$. Our estimate for $\Delta \omega$ assumes that rotation lifts $m$ degeneracy. If it does not, the minimum frequency mismatch is increased by a factor of $\ell_{d}$.

Next we describe how $\gamma$ varies with $\omega$ and $\ell$. There are two regimes of relevance to this investigation. In the quasiadiabatic limit (see $\S 4.4$ of Paper I), ${ }^{7}$

$$
\gamma \sim \frac{1}{n \tau_{\omega}} \propto\left(\frac{\ell}{\omega}\right)^{6} .
$$

In the strongly nonadiabatic limit (see $\S \mathrm{B} 1$ ),

$$
\gamma \sim \frac{\omega}{\pi n} \ln \frac{1}{\mathscr{R}} \propto \omega^{0.75} \ell^{0.2},
$$

where $\mathscr{R}$ is the amplitude reflection coefficient at the top of the mode's cavity. The transition between the quasiadiabatic and strongly nonadiabatic limits is marked by a significant reduction of $\mathscr{R}$ by radiative diffusion. The behavior of $\gamma$ as a function of $\omega$ and $\ell$ is illustrated in Figure 5.

\footnotetext{
${ }^{6}$ The factor $n_{p}$ arises because the peak in $\kappa$ has a width $\left|n_{d_{1}}-n_{d_{2}}\right| \sim$ $n_{p \cdot}{ }_{7}$ Overstable modes are quasi-adiabatic, so this estimate for $\gamma$ applies to them as well as to damped modes.
} 

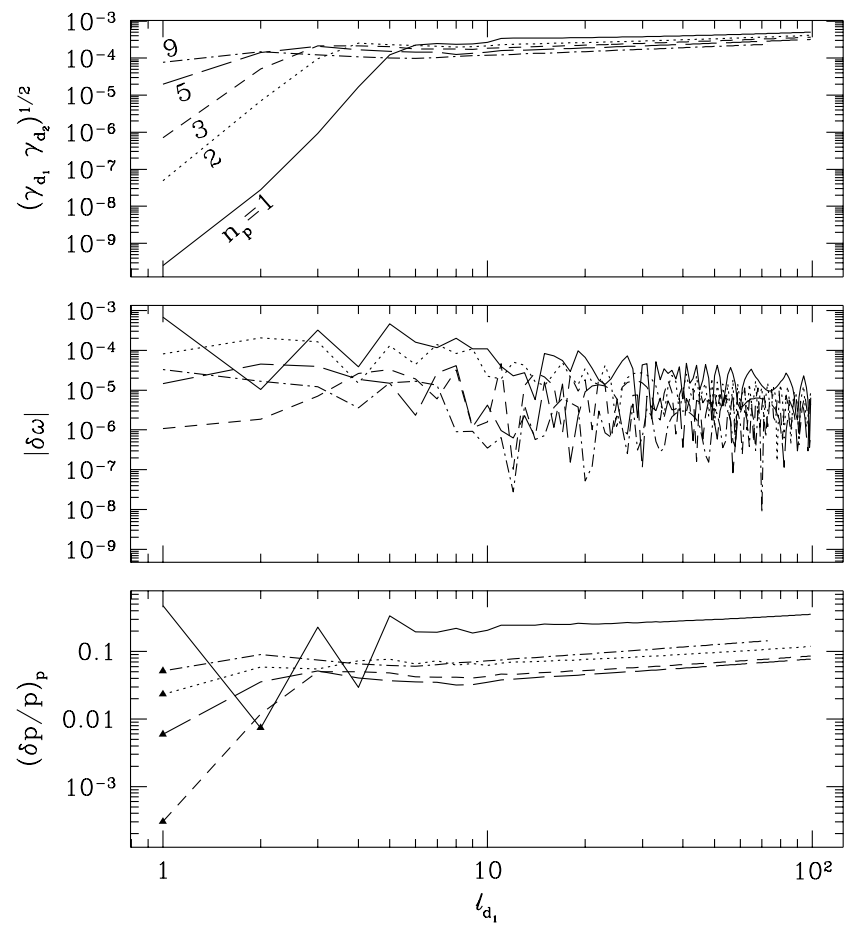

FIG. 6.-Selection of the best daughter pairs for a few low-order $\ell_{p}=1$ parent modes in a white dwarf model with $T_{\text {eff }}=12,800 \mathrm{~K}$. Results for individual $n_{p}$ are distinguished by different line styles as defined in the upper panel. We look at daughter pairs with $\ell_{d_{1}}$ ranging from 1 to 100 and $\ell_{d_{2}}=\ell_{d_{1}}+\ell_{p}$. We plot $\left(\gamma_{d_{1}} \gamma_{d_{2}}\right)^{1 / 2}$ in the upper panel and $|\delta \omega|$ in the middle panel for the pair that sets the lowest instability threshold at each $\ell_{d_{1}}$. This does not necessarily correspond to the pair that gives rise to the lowest $|\delta \omega|$ at each $\ell_{d_{1}}$. The corresponding threshold value of $(\delta p / p)_{p}$ evaluated at the stellar surface is shown in the bottom panel. Except for very low order parent modes, the best daughter pairs satisfy $\ell_{d_{1}}=1$ and $|\delta \omega|<\gamma_{d}$.

For $n_{p} \leq 3$, minimal $\left(\delta \omega^{2}+\gamma_{d}^{2}\right)$ is achieved with $\ell_{d_{1}}$ greater than unity (see Fig. 6), whereas for $n_{p} \geq 5$, the best daughter pairs have $\ell_{d_{1}}=1$.

\section{UPPER ENVELOPE OF PARENT MODE AMPLITUDES}

Parametric instability provides an upper envelope to the amplitudes of overstable modes. Coupling of an overstable parent mode to a single pair of daughter modes suffices to maintain the parent mode's amplitude near the threshold value (see $\S 2.2$ ). The energy gained by the overstable parent mode and transferred to the daughter modes may be disposed of by linear radiative damping or by further nonlinear coupling to granddaughter modes.

The results presented in this section are obtained from numerical computations and displayed in a series of figures. The general trends they exhibit are best understood in terms of analytic scaling relations. We derive these first in order to be able to refer to them as we describe each figure.

Excited modes of ZZ Ceti stars are usually detected through photometric measurements of flux variations and in a few cases through spectroscopic measurements of horizontal velocity variations. Hence, we calculate surface amplitudes of fractional flux $(\Delta F / F)$ and horizontal velocity $\left(v_{h}\right)$ variations. ${ }^{8}$ Each of these is directly related to the near surface amplitude of the Lagrangian pressure perturbation,

\footnotetext{
${ }^{8}$ In this section we drop subscripts on parent mode parameters and denote daughter mode parameters by a subscript $d$.
}

$\delta p / p$. The mode energy is related to the surface value of $(\delta p / p)^{2}$ by the normalization factor given in equation (A28) of Paper I. Thus,

$$
E \sim \frac{1}{n \tau_{\omega} L}\left(\frac{\delta p}{p}\right)^{2}
$$

It follows that

$$
\frac{\delta p}{p} \sim n\left[\left(\frac{\gamma_{d}}{\omega}\right)^{2}+\left(\frac{\delta \omega}{\omega}\right)^{2}\right]^{1 / 2}
$$

where the weak dependence on the properties of the parent mode is due to the cancellation of $n \tau_{\omega}$ between $\kappa$ and the normalization factor. Adopting relations expressing $v_{h}$ and $\Delta F / F$ in terms of $\delta p / p$ from $\S 3$ of Paper I, we arrive at

$$
v_{h} \sim \frac{\omega R n}{[\ell(\ell+1)]^{1 / 2}}\left[\left(\frac{\gamma_{d}}{\omega}\right)^{2}+\left(\frac{\delta \omega}{\omega}\right)^{2}\right]^{1 / 2}
$$

and

$$
\frac{\Delta F}{F} \sim \frac{n}{\left[1+\left(\omega \tau_{c}\right)^{2}\right]^{1 / 2}}\left[\left(\frac{\gamma_{d}}{\omega}\right)^{2}+\left(\frac{\delta \omega}{\omega}\right)^{2}\right]^{1 / 2} .
$$

In the above, $R$ denotes the stellar radius and $\tau_{c}$ is the thermal time constant describing the low-pass filtering action of the convection zone on flux variations input at its base. $^{9}$

Figure 7 displays calculated values for amplitudes of overstable modes limited by parametric instability. Amplitudes of $|\delta p / p|$ generally rise with increasing mode period, except for the shortest period modes, which exhibit large star-to-star fluctuations. As indicated by equation (17), this rise mainly reflects the corresponding rise of the damping rates of the daughter modes. The amplitudes for $\ell=1$ modes in the coolest model dip around $600 \mathrm{~s}$ and flatten beyond $1200 \mathrm{~s}$. The first feature is associated with the dip in $\gamma$ for neutrally stable daughter modes, and the second is explained by the flattening of $\gamma$ for strongly nonadiabatic daughter modes of long period (see Fig. 5). At the longer periods, the values of $|\delta p / p|$ decline with decreasing $T_{\text {eff }}$. This is a subtle consequence of the deepening of the convection zone, which pushes down the top of the daughter modes' cavities, thus reducing their damping rates (see $\S 5.2$ of Wu \& Goldreich 1999, hereafter Paper II). The behavior of $\left|v_{h}\right|$ is similar to that of $|\delta p / p|$ except that $\left|v_{h}\right|$ decreases relative to $|\delta p / p|$ with increasing $\ell$ as shown by comparison of equations (17) and (18). A new feature present in the run of $|\delta F / F|$ versus mode period is the low-pass filtering action of the convection zone as expressed by the factor $\left[1+\left(\omega \tau_{c}\right)^{2}\right]^{-1 / 2}$ in equation (19). This factor causes $|\delta F / F|$ to rise slightly more steeply than $|\delta p / p|$ with increasing mode period. It is also responsible for a more dramatic decrease in $|\delta F / F|$ with decreasing $T_{\text {eff }}$ at fixed mode period. Since $\left|v_{h}\right|$ does not suffer from this visibility reduction, velocity variations may be observable in stars that are cooler than those at the red edge of the instability strip. Nonadiabaticity reduces the couplings between long-period parent modes and their daughters (Appendix B, § B2). Consequently, threshold amplitudes for these modes should be slightly higher than those plotted in the figure.

\footnotetext{
${ }^{9}$ Here $\tau_{c} \approx 3 \tau_{\text {th }}$, where $\tau_{\text {th }}$ is the conventional thermal relaxation time evaluated at $z_{b}$, the bottom of the surface convection zone.
} 

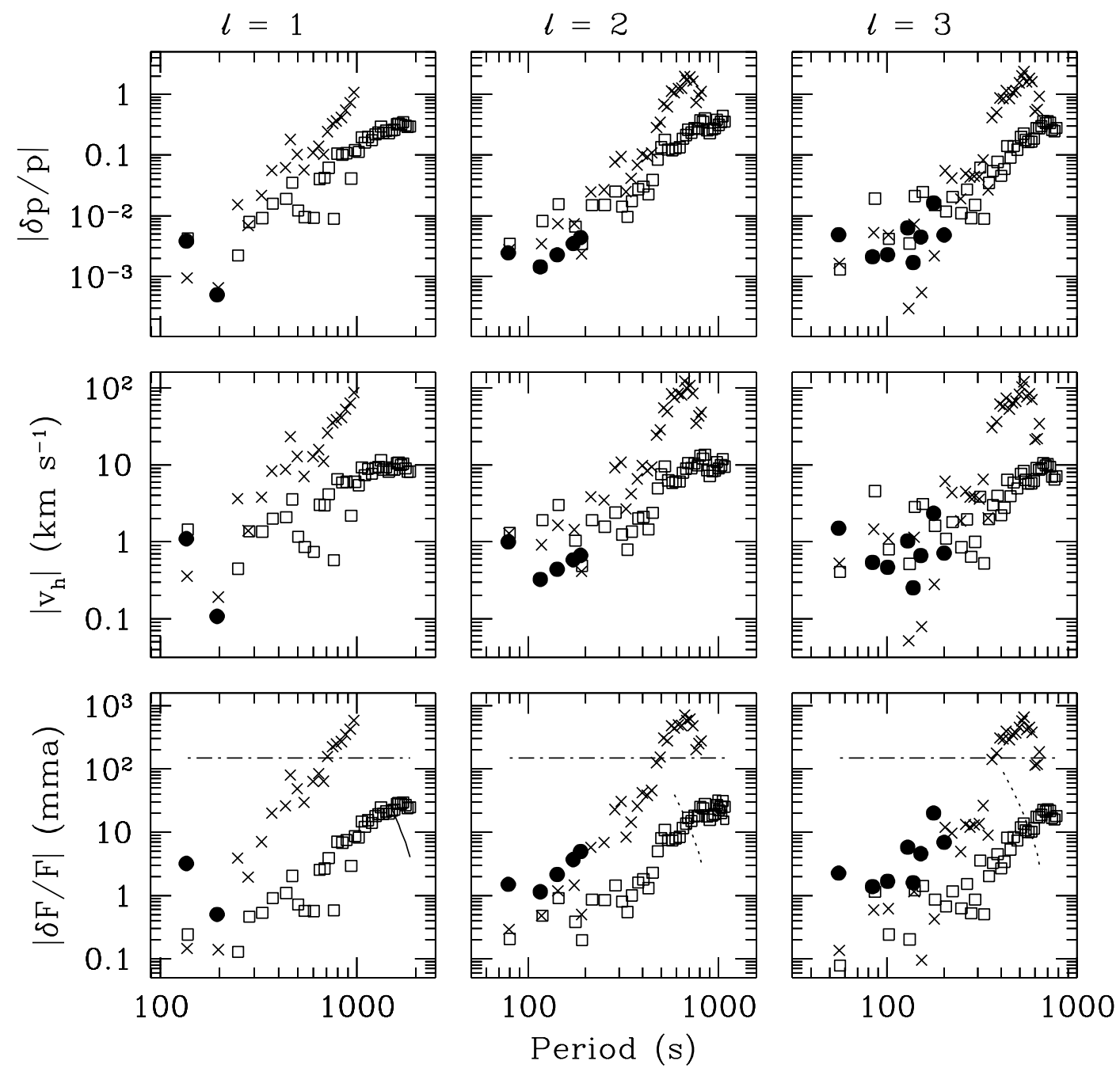

Fig. 7.-An assembly of results on threshold amplitudes for parametric instability. Parent-daughter pairs are subject to the constraint that $\gamma_{d} \geq 4 \gamma_{p}$. From left to right, amplitudes of overstable modes with different $\ell$ are plotted against mode period. From top to bottom, the panels display photospheric amplitudes of fractional pressure perturbation, horizontal velocity, and fractional flux variation. Although mode coupling coefficients used in the determination of parametric instability thresholds are calculated using adiabatic eigenfunctions, photospheric values of $|\delta p / p|,\left|v_{h}\right|$, and $|\delta F / F|$ are obtained using nonadiabatic eigenfunctions. Filled circles, crosses, and open squares symbolize modes from the three stellar models in Fig. 5 in order of descending $T_{\text {eff }}$. Turbulent dissipation arising from the shear layer below the convection zone may suppress the amplitudes that long-period modes can attain (§ 5.1). This is illustrated, where appropriate, in the lower panels by a short dotted line for the medium temperature model and a solid line for the coolest model. We have taken $C_{D}=5 \times 10^{-3}$ (Jensen, Sumer, \& Fredsoe 1989). When the surface flux amplitude rises above $\sim 15 \%$, the convection zone evaporates during part of the cycle. This is marked by the dot-dashed horizontal lines in the lower panels.

Figure 8 reproduces a summary of observational data on mode amplitudes from Clemens (1995). Each variable is represented by a star showing the relation between the optical photometric amplitude in its largest mode and the powerweighted mean period of all its observed modes. The latter quantity is a surrogate for the star's effective temperature in the sense that longer mean periods correspond to lower effective temperatures (Clemens 1995; Paper I). Our theoretical predictions for the amplitudes of $\ell=1$ modes are shown by the filled circles. Although based on only three models, our calculations exhibit an encouraging similarity to the observed mode amplitudes including their dependence on mode period.

For a few low-order overstable modes, $n_{p} \leq 3$ at $\ell_{p}=1$, $\delta \omega$ is more significant than $\gamma_{d}$ in determining the best daughter pair (Fig. 6). The best daughter pairs for these modes have $\ell$ values of a few and identities that depend sensitively on minor differences among stars. Thus, we expect amplitudes of short-period overstable modes to show large star-to-star variations. That the $n=1, \ell=1$ mode is detected in only about one-half of the hot DAVs (see Fig. 4 of Clemens 1995) is consistent with the expected statistical variations in $\delta \omega$.

Overstable $\ell=1$ modes with periods longer than $1000 \mathrm{~s}$ are probably not saturated by parametric instability. Their maximum $|\kappa|$ is severely reduced below the adiabatic value by the strong nonadiabaticity of their daughter modes ( $\$ \mathrm{~B} 2)$. We include the effects of turbulent saturation on these modes in Figure 8 and discuss it in $\S 5$. Effects arising from the nonlinear saturation of daughter mode amplitudes are also discussed there.

\section{DISCUSSION}

\subsection{Turbulent Saturation of the Parent Modes}

Turbulent viscosity severely reduces the vertical gradient 


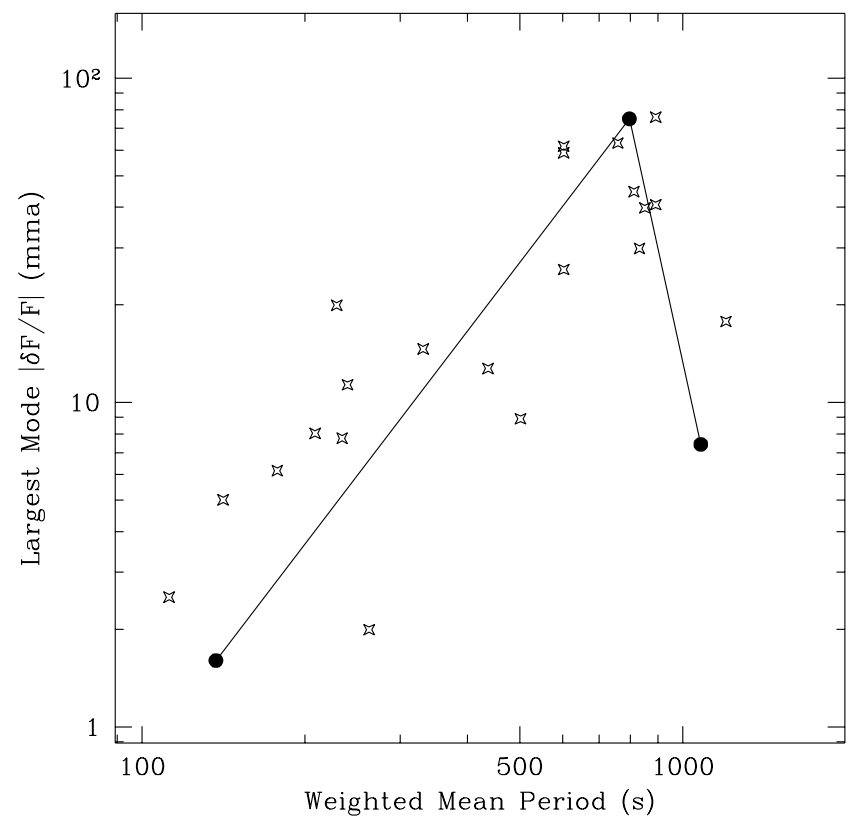

FIG. 8.-Comparison between theoretical parametric amplitudes and those summarized by Clemens (1993) from observations of two dozen DA variables. Each variable is represented by an open star plotted such that the ordinate gives the photometric amplitude of the largest mode and the abscissa the mean period weighted by the power (square of the photometric amplitudes) of all its detected modes. To compare theory and observation, we extract the amplitude of the largest mode and compute the power-weighted mean period for each of the stellar models considered in Fig. 7. This procedure is carried out for $\ell=1$ modes subject to the constraint that $\delta F / F \leq 15 \%$ and accounting for saturation by nonlinear turbulent damping based on the choice $C_{D}=5 \times 10^{-3}(\S 5.1)$. The latter only affects the amplitudes of modes with periods greater than $1000 \mathrm{~s}$. The filled circles display our theoretical results, with the calculated values of $|\delta F / F|$ reduced by a factor of 2 to account approximately for the reduction in optical relative to bolometric flux variations for DA variables. We expect large star-to-star variations associated with different values of frequency mismatches in the short-period end. The drop-off in amplitude at long period $\left(P>10^{3} \mathrm{~s}\right)$ results from a combination of lower daughter mode damping rates, a reduction in the visibility of the parent mode due to increasing $\omega \tau_{c}$, and turbulent saturation.

of the horizontal velocity of $g$-modes in the convection zone (Brickhill 1990). As a result, a shear layer forms at the boundary between the bottom of the convection zone and the top of the radiative interior (Goldreich \& Wu 1999, hereafter Paper III). Kelvin-Helmholtz instability of this shear layer provides a potential source of nonlinear mode damping.

The surface amplitude of $(\delta p / p)$ at which nonlinear turbulent damping balances linear convective driving is given by equation (44) of Paper III:

$$
\left(\frac{\delta p}{p}\right)_{\mathrm{turb}} \sim \frac{0.1}{C_{D}} \frac{\left[\left(\omega \tau_{c}\right)^{2}+1\right]^{1 / 2}\left[\left(\omega \tau_{c}\right)^{2}-1\right]}{\omega \tau_{c}} \frac{k_{h} z_{\omega}^{2}}{z_{b}},
$$

where $C_{D}$ is the dimensionless drag coefficient and $k_{h}$ is the horizontal wavevector. This amplitude drops sharply with increasing mode period.

It might appear from equation (20) that nonlinear turbulent damping could enforce an arbitrarily small mode amplitude. This is incorrect. The finite mass of the convection zone sets an upper limit on the turbulent stress (eq. [41] of Paper III) that can act on the convection zone. It cannot exceed the value at which the convection zone is dragged along with the top of the radiative interior. This limit translates into

$$
C_{D} \rho_{b}\left|\Delta v_{h}\right|^{2} \sim \rho_{b} \omega\left|\Delta v_{h}\right|,
$$

where $\rho_{b}$ is the density at $z_{b}$ and $\Delta v_{h}$ is the velocity jump across the shear layer. Thus, turbulent saturation is ineffective for modes with $\left|\Delta v_{h}\right| \gtrsim \omega z_{b} / C_{D}$. It is more revealing to express this condition in terms of the maximum surface value of $(\delta p / p)$ at which turbulent saturation is effective. By use of equation (39) of Paper III, we obtain

$$
\left(\frac{\delta p}{p}\right)_{\max } \sim \frac{1}{C_{D}}\left[1+\left(\omega \tau_{c}\right)^{2}\right]^{1 / 2} k_{h} z_{\omega} .
$$

Comparison of equations (20) and (22) demonstrates that nonlinear turbulent damping cannot saturate the amplitudes of modes with $z_{\omega} \gg z_{b}$.

Very long period overstable modes $(P>1000$ s) satisfy $z_{\omega} \sim z_{b}$. Turbulent saturation may be important for them as illustrated in Figure 7.

\subsection{Nonlinear Damping of Daughter Modes \\ 5.2.1. Granddaughter Modes}

Here we answer the following questions. Under what conditions do daughter modes excite granddaughter modes by parametric instability ${ }^{10}$ What are the consequences if they do? In $\S 2.2$ we show how parametric instability of linearly damped daughter modes maintains the amplitude of a parent mode close to its equilibrium value. In order to dispose of the energy they receive from the parent mode, the time-averaged energies of the daughter modes must be close to their equilibrium values. This raises a worry. Suppose the daughters are prevented from reaching their equilibrium amplitudes by parametric instability of granddaughter modes. Then they would not be able to halt the amplitude growth of the parent mode.

To answer the first of these questions, we calculate the ratio, denoted by the symbol $\mathscr{S}$, between the threshold amplitude for a daughter mode to excite granddaughter modes and its equilibrium amplitude under parametric excitation by the parent mode. The former is obtained from equation (4), and the latter from equations (6) and (7). We make a few simplifying assumptions to streamline the discussion. Resonances between daughters and granddaughters are taken as exact; individual members of daughter and granddaughter pairs are treated as equivalent. Equations (12) and (14) are combined to yield

$$
\kappa^{2} \sim \frac{\gamma_{p}}{n_{p}^{2} L} .
$$

It is then straightforward to show that

$$
\mathscr{S} \approx \frac{\omega_{p} \omega_{d}}{\omega_{g}^{2}}\left(\frac{n_{d}}{n_{p}}\right)^{2}\left(\frac{\gamma_{g}^{2}}{\gamma_{d}^{2}+\delta \omega^{2}}\right) \approx 32\left(\frac{\gamma_{g}^{2}}{\gamma_{d}^{2}+\delta \omega^{2}}\right) .
$$

The factor 32 is an approximation based on taking $\omega_{g} / \omega_{d}=$ $\omega_{d} / \omega_{p}=\frac{1}{2}$ and $n_{p} / n_{d}=\frac{1}{2}$.

In general $\mathscr{S} \gg 1$, so the excitation of granddaughter modes requires the daughter modes to have energies in excess of their equilibrium values. However, the equilibrium solution is unstable if the best daughter pair corresponds to

\footnotetext{
${ }^{10}$ In this subsection subscripts $p, d$, and $g$ refer to parent, daughter, and granddaughter modes.
} 
$\gamma_{d}>|\delta \omega|$, and then the daughter mode energies episodically rise far above their equilibrium values. At such times, granddaughter modes may be excited by parametric instability and consequentially limit the amplitude growth of the daughter modes. This slows the transfer of energy from parent to daughter modes, but it does not prevent the daughter modes from saturating the growth of the parent mode's amplitude at the level described by equation (4).

For the few lowest order parent modes, we typically find $|\delta \omega| \geq \gamma_{d}$. This may reduce $\mathscr{S}$ to below unity with the consequence that the parent mode amplitude may rise above that given by equation (4).

\subsubsection{Turbulent Saturation of Daughter Mode Amplitudes}

As the parent mode's equilibrium amplitude is determined by the rate of total dissipation in the daughter modes, we consider here the importance of turbulent dissipation for the latter modes.

An argument almost identical to that given in $\S 5.1$ establishes that nonlinear turbulent damping cannot exceed linear damping for daughter modes with $z_{\omega}>z_{b}$. However, daughter modes with $z_{\omega} \ll z_{b}$ might suffer severe nonlinear turbulent dissipation. But as these modes propagate immediately below the convection zone, they undergo strong nonadiabatic linear damping with a rate (see eq. [B4])

$$
\gamma \approx \frac{\omega}{\pi n} \ln \mathscr{R}^{-1}
$$

where $\mathscr{R}$ is the reflection coefficient at the top of the mode cavity. Strong radiative diffusion implies $\mathscr{R} \ll 1$, and turbulent dissipation further reduces it. However, the effective dissipation rate likely remains close to $\omega / \pi n$. We conclude that it is safe to neglect the nonlinear turbulent damping of daughter modes.

\subsection{Additional Three-Mode Interactions}

Parametric instability sets reasonable upper bounds on the photospheric amplitudes of overstable modes. These upper bounds vary smoothly with mode period. However, the observed amplitude distributions are highly irregular. This mode selectivity may arise from three-mode interactions, which involve more than one overstable mode.

We investigate a particular example of this type. It is closely related to parametric instability, the only difference being that the daughter modes of the overstable parent mode are themselves overstable. Acting in isolation, resonant mode couplings tend to drive mode energies toward equipartition. They conserve the total energy, $\dot{E}_{p}+\dot{E}_{d_{1}}$ $+\dot{E}_{d_{2}}=0$, and transfer action according to $\dot{E}_{d_{1}} / \omega_{d_{1}}=$ $\dot{E}_{d_{2}} / \omega_{d_{2}}=-\dot{E}_{p} / \omega_{p}$. In this context it is important to note that the energies of overstable modes limited by parametric instability decline with increasing mode period (Fig. 9). Therefore, nonlinear interactions transfer energy from the parent mode to its independently excited daughters. As shown below, this transfer may severely suppress the parent mode's amplitude.

We start from equations (1)-(3). These may be manipulated to yield

$$
\frac{d E_{p}}{d t}=\gamma_{p} E_{p}+3 \sqrt{2} \omega_{p} \kappa\left(E_{p} E_{d_{1}} E_{d_{2}}\right)^{1 / 2} \sin \Phi,
$$

where $\Phi=\theta_{d_{1}}+\theta_{d_{2}}-\theta_{p}$. For $E_{p} \gg E_{d_{1}}$ and $E_{d_{2}}$, nonlinear interactions transfer energy from the parent mode to its

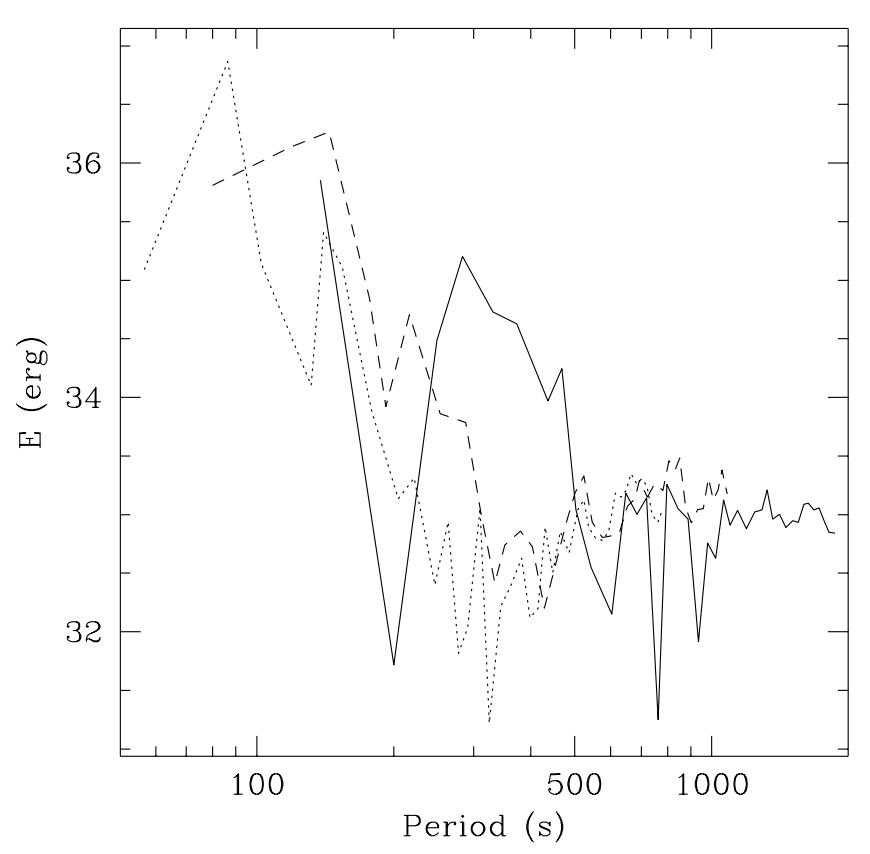

Fig. 9.- Mode energy vs. period for overstable modes in the coolest model considered in Fig. 7. Solid, dashed, and dotted lines represent $\ell=1$, 2 , and 3 modes. Mode energy tends to decrease with increasing period and with increasing $\ell$. Deviations from this trend for the $\ell=1$ modes are explained as follows. The narrow dip near $200 \mathrm{~s}$ is due to a fortuitously good frequency resonance for the $n=2$ mode, and the wider dip around $700 \mathrm{~s}$ is associated with neutrally stable daughter modes.

daughter modes. In particular, if we ignore phase changes in the overstable daughter modes due to their interactions with granddaughter modes, we find that $\Phi$ satisfies

$$
\begin{aligned}
\frac{d \Phi}{d t}= & \delta \omega-\frac{3}{\sqrt{2}} \kappa\left(E_{p} E_{d_{1}} E_{d_{2}}\right)^{1 / 2} \\
& \times\left[\left(\frac{\omega_{d_{1}}}{E_{d_{1}}}+\frac{\omega_{d_{2}}}{E_{d_{2}}}\right)-\frac{\omega_{p}}{E_{p}}\right] \cos \Phi,
\end{aligned}
$$

with a stable solution at $\Phi=-\pi / 2$ when $\delta \omega=0$.

We denote the ratio of the nonlinear term to the linear term in equation (26) by the symbol $\mathscr{T}$ :

$$
\mathscr{T} \approx \frac{3 \sqrt{2} \omega_{p} \kappa}{\gamma_{p}}\left(\frac{E_{d_{1}} E_{d_{2}}}{E_{p}}\right)^{1 / 2} .
$$

Using the magnitudes of $E_{i}$ set by parametric instability of their respective daughters and adopting the same approximations made in $\S 5.2$, we arrive at

$$
\mathscr{T} \approx \frac{\omega_{p} \omega_{d}}{\omega_{g}^{2}}\left(\frac{n_{d}}{n_{p}}\right)^{2}\left(\frac{\gamma_{g}^{2}}{\gamma_{d}^{2}+\delta \omega^{2}}\right) \approx 32\left(\frac{\gamma_{g}^{2}}{\gamma_{d}^{2}+\delta \omega^{2}}\right) .
$$

Comparing equations (24) and (29), we see that $\mathscr{S}=\mathscr{T}$. A little thought reveals that this is not a coincidence. For $\mathscr{T} \gg 1$, overstable daughter modes can suppress a parent mode's energy below the value set by parametric instability. We expect this suppression to be important in cool ZZ Ceti stars whose overstable modes extend to long periods. It may render their intermediate-period modes invisible. In a similar manner, the amplitudes of high-frequency overstable modes with $\ell=2$ and 3 may be heavily suppressed by interactions with their lowest $\ell$ overstable daughters. 
The irregular amplitude distributions among neighboring modes may be partially accounted for by this type of resonance. Mode variability may also play a role. We explore this in the next subsection.

\subsection{Mode Variability}

Some excited $g$-modes in ZZ Ceti stars are exemplary clocks, while others exhibit substantial temporal variations. Parametric instability may at least partially account for these different behaviors.

Kepler et al. (2000) find a period drift rate of $\dot{p} / p \sim 10^{-17}$ $\mathrm{s}^{-1}$ for the $215 \mathrm{~s}$ mode in DA G117-B15A. The linear growth rate for this mode is much larger than $10^{-17} \mathrm{~s}^{-1}$. It is plausible that the amplitude and the phase of this mode are stabilized by parametric interaction with a daughter pair for which $\delta \omega>\gamma_{d}(\operatorname{see} \S 2.2)$.

We also show in $\S 2.2$ that for $|\delta \omega|<\gamma_{d}$, parametric instability gives rise to limit cycles in which the amplitudes and phases of parent and daughter modes vary on timescales as short as $\gamma_{d}^{-1}$. During these cycles, flux variations associated with stable daughter modes may occasionally exceed those of their parent modes. Temporal amplitude variations may also contribute to the irregular mode amplitude distribution seen in individual stars.

Phase variations of a parent mode obey the equation

$$
\frac{d \theta_{p}}{d t}=\omega_{p}-\frac{3}{\sqrt{2}} \omega_{p} \kappa \frac{\left|A_{d_{1}}\right|\left|A_{d_{2}}\right|}{\left|A_{p}\right|} \cos \Phi .
$$

At the equilibrium given by equations (5)-(8), the parent mode's frequency is displaced from its unperturbed value such that

$$
\begin{gathered}
\omega_{p}^{\prime}=\frac{d \theta_{p}}{d t}=\omega_{p}+\frac{\delta \omega \gamma_{p}}{\gamma_{d_{1}}+\gamma_{d_{2}}-\gamma_{p}} \\
\times \sqrt{1+\left(\frac{2 \delta \omega}{\gamma_{d_{1}}+\gamma_{d_{2}}-\gamma_{p}}\right)^{2}} .
\end{gathered}
$$

This constant frequency shift is of order $10^{-9} \mathrm{~s}^{-1}$ for the $n=1, \ell=1$ mode and of order $10^{-7} \mathrm{~s}^{-1}$ for the $n=2$, $\ell=2$ mode. Frequency shifts in higher order overstable modes that are involved in limit cycles are predicted to be larger and time variable. During brief intervals of length $\sim \gamma_{d}^{-1}$, when the daughter mode energies are comparable to that of the parent mode, $\left|\omega_{p}^{\prime}-\omega_{p}\right| \sim \gamma_{d}$. These frequency shifts can be as large as a few microhertz in angular frequency. Such shifts might account for the time-varying rotational splittings reported by Kleinman et al. (1998) provided that different $m$ components of the overstable modes are involved in different limit cycles.

In circumstances of small rotational splitting, the simple limit cycles depicted in Figure 1 are unlikely to be realistic. In such cases, different $m$ components of an overstable parent mode share some common daughter modes. This leads to more complex dynamics.

\subsection{Mode Trapping}

Gravitational settling produces chemically pure layers between which modes can be partially trapped. Consider a parent mode that is trapped in the hydrogen layer. Trapping enhances its normalization factor and $|\kappa|_{\max }$ by identical amounts relative to the values these quantities have for untrapped modes of similar frequency (see Appendix A, $\S$
A2). This implies that trapping does not affect the predicted photospheric amplitudes of $\delta p / p$, and hence $v_{h}$ and $\delta F / F$, since these are proportional to $A$ times the square root of the mode normalization factor. However, trapping does lower the threshold energy for parametric instability.

Photospheric amplitudes of parent modes are insensitive to mode trapping under quite general circumstances because all of the significant driving and nonlinear damping takes place in the parent mode's upper evanescent layer. Trapping only alters the relative magnitude of the parent mode's eigenfunction in different regions. It does not alter the equilibrium values for the surface perturbations at which damping balances driving.

\section{SUMMARY}

\subsection{Current Status}

The current paper is the fourth in our series on ZZ Ceti stars. To place it in perspective, we open this section by offering our views on what has been achieved and what remains elusive.

We have a sound analytic understanding of linear damping and driving for modes that satisfy both $z_{\omega} \gg z_{b}$ and $\omega \tau_{c} \gg 1$ (Paper I). In particular, convective driving as proposed by Brickhill (1991) is responsible for mode excitation. Fully nonadiabatic numerical computations (Paper II) extend the linear analysis to modes with arbitrary values of $z_{\omega}$ and $\omega \tau_{c}$.

Lack of knowledge about convective overshoot in the top of the radiative layer frustrates our attempts to evaluate linear driving and damping for modes with $z_{\omega} \sim z_{b}$. Convective overshoot enhances linear damping due to both radiative diffusion and turbulent viscosity (Paper II; Paper III). Unless we include it, our numerical calculations predict that periods of overstable modes extend up to $2400 \mathrm{~s}$, twice as long as the maximum observed. More than a scale height of overshoot is required to reduce the calculated upper limit to $1200 \mathrm{~s}$.

Kelvin-Helmholtz instability (Paper III) of the modedriven shear layer below the convection zone may affect mode amplitudes and variability. Three conditions are necessary for shear instability to occur. The shear rate must exceed both the mode frequency and the buoyancy frequency, and the Reynolds number based on the turbulent viscosity associated with overshoot must be large compared to unity. While the first and third conditions are easily met by modes with amplitudes comparable to those observed (Paper III), the situation for the second condition is less clear. Stable stratification in the overshoot layer is set by a balance between turbulent mixing, which reduces stability, and radiative diffusion, which enhances it. Without a detailed understanding of the overshoot region, something that remains elusive even for the Sun, we are unable to determine the importance of the shear instability.

\subsection{Conclusions}

Mode coupling is likely to be of relevance to amplitude saturation and variability of overstable modes in smallamplitude, multimode pulsators like ZZ Ceti stars. Parametric instability is a specific example of mode coupling. It results in the transfer of energy from an overstable parent mode to one or more pairs of daughter modes. Provided the daughter modes can dispose of this energy, parametric instability limits the time-averaged amplitude the parent mode can attain. 
We demonstrate that parametric instability is capable of saturating the amplitudes of overstable $g$-modes in ZZ Ceti stars. Amplitudes we calculate for flux variations are similar to those observed. They tend to be larger for longer period modes in accord with observation.

Based on our calculations, saturation of high-order modes $\left(z_{\omega} \sim z_{b}\right)$ by parametric instability occurs at amplitudes $|\delta F / F| \gtrsim 15 \%$. There are two reasons why these calculated amplitudes may be excessive. Kelvin-Helmholtz instability may saturate the amplitudes of high-order modes at smaller values. At the calculated amplitudes, a major fraction of the convective region is evaporated during part of the pulsation cycle. This is likely to reduce convective driving, something our calculations do not account for.

Parametric instability leads to a stable equilibrium state if $|\delta \omega|>\gamma_{d}$ and to a limit cycle if $|\delta \omega|<\gamma_{d}$. For parent modes with $\ell_{p}=1$, the former applies if $n_{p} \leq 3$ and the latter if $n_{p} \geq 5$.

Detected modes probably include damped daughters as well as overstable parents. In the case of stable equilibrium, surface flux variations due to daughters are comparable to that of their parent. However, the daughters are likely to have $\ell_{d}>1$ and thus be more difficult to detect. Surface flux variations due to daughters involved in a limit cycle, while smaller than that of their parent over most of the cycle, peak at larger values. Moreover, visibility is less of an issue in this case since we expect $\ell_{d_{1}}=1$.

Based on our predictions of mode amplitudes, we suspect that excited modes may be discovered below the red edge by detection of their photospheric velocity variations. The absence of a firm prediction for the red edge of the instability strip is perhaps the most glaring shortcoming of our investigations to date.

We are indebted to P. Bradley for supplying us with models of DA white dwarfs and to an anonymous referee for comments that greatly improved our article. Financial support for this research was provided by NSF grant 9414232 .

\section{APPENDIX A}

\section{THREE-MODE COUPLING COEFFICIENT}

We reproduce the expression for the three-mode coupling coefficient presented in equation (9) of $\S 3.1$ with one modification:

$$
\kappa=-\int d^{3} x p\left[\frac{\Gamma_{1}\left(\Gamma_{1}-2\right)}{6}(\nabla \cdot \xi)^{3}+\frac{1}{2} \Gamma_{1}(\nabla \cdot \xi) \xi_{; j}^{i} \xi_{; i}^{j}+\operatorname{det}\left|\xi_{; j}^{i}\right|\right]
$$

where

$$
\operatorname{det}\left|\xi_{; j}^{i}\right|=\frac{1}{6}(\nabla \cdot \xi)^{3}-\frac{1}{2}(\nabla \cdot \xi) \xi_{; j}^{i} \xi_{; i}^{j}+\frac{1}{3} \xi_{; j}^{i} \xi_{; k}^{j} \xi_{; i}^{k} .
$$

Because of strong cancellations among its largest terms, this form is not well suited for numerical evaluation. We derive a new expression that does not suffer from this defect. Then we estimate the size of $\kappa$ and deduce its dependences upon the properties of the three modes.

\section{A1. SIMPLIFICATION}

In spherical coordinates $(r, \theta, \phi)$, the components of the displacement vector may be written as

$$
\boldsymbol{\xi}=\xi^{i} \boldsymbol{\varepsilon}_{i}=\left[\xi_{r}(r), \frac{\xi_{h}(r)}{r} \frac{\partial}{\partial \theta}, \frac{\xi_{h}(r)}{r} \frac{1}{\sin ^{2} \theta} \frac{\partial}{\partial \phi}\right] Y_{\ell m}(\theta, \phi),
$$

where $Y_{\ell m}$ is a spherical harmonic function and the $\varepsilon_{i}$ are covariant basis vectors.

The angular integrations in equation (A1) are done analytically. The following definitions and properties prove useful (see Kumar \& Goodman 1996):

$$
\begin{aligned}
T & \equiv \int_{0}^{2 \pi} d \phi \int_{0}^{\pi} d \theta \sin \theta Y_{a} Y_{b} Y_{c} \equiv\left\langle Y_{a} Y_{b} Y_{c}\right\rangle, \\
F_{a} & \equiv\left\langle Y_{a} \nabla_{\mu} Y_{b} \nabla^{\mu} Y_{c}\right\rangle=\left(\Lambda_{b}^{2}+\Lambda_{c}^{2}-\Lambda_{a}^{2}\right) \frac{T}{2}, \\
G_{a} & \equiv\left\langle\nabla_{\mu} \nabla_{v} Y_{a} \nabla^{\mu} Y_{b} \nabla^{v} Y_{c}\right\rangle \equiv\left[\Lambda_{a}^{4}-\left(\Lambda_{b}^{2}-\Lambda_{c}^{2}\right)^{2}\right] \frac{T}{4}, \\
S & \equiv\left\langle\nabla_{\mu} \nabla^{v} Y_{a} \nabla_{v} Y_{b} \nabla^{\mu} Y_{c} \nabla_{\mu} \nabla^{\mu} Y_{a} \nabla_{v} Y_{b} \nabla^{v} Y_{c}\right\rangle=G_{a}+G_{b}+G_{c}=\frac{1}{2}\left(\Lambda_{a}^{2} F_{a}+\Lambda_{b}^{2} F_{b}+\Lambda_{c}^{2} F_{c}\right)=G_{a}+\Lambda_{a}^{2} F_{a}, \\
V_{a} & \equiv\left\langle Y_{a} \nabla_{\mu} \nabla^{v} Y_{b} \nabla_{v} \nabla^{\mu} Y_{c}\right\rangle=\Lambda_{b}^{2} \Lambda_{c}^{2} T-F_{a}-S, \\
\Lambda_{a}^{2} T & =F_{b}+F_{c}, \\
\Lambda_{a}^{2} F_{a} & =G_{b}+G_{c} .
\end{aligned}
$$


Here $\nabla_{\mu}$ is the covariant derivative on a spherical surface; $\mu$ can be either $\theta$ or $\phi$. Each angular integration is proportional to $T$, which contains all $m$ dependences and is of order unity independent of the $\ell$ values of the participating modes. The parameter $\Lambda^{2} \equiv \ell(\ell+1)$. Subscripts $a, b$, and $c$ denote different modes. The angular selection rules are simply $\ell_{c} \in\left[\left|\ell_{a}-\ell_{b}\right|\right.$, $\left.\ell_{a}+\ell_{b}\right], \bmod \left[\left(\ell_{a}+\ell_{b}+\ell_{c}\right), 2\right]=0$, and $m_{a}+m_{b}+m_{c}=0$.

Angular integration of det $\left|\xi_{; j}^{i}\right|$ yields

$$
\oint d \Omega \operatorname{det}\left|\xi_{; j}^{i}\right|=\frac{1}{r^{2}}\left[T \xi_{r}^{b} \xi_{r}^{c}-\Lambda_{c}^{2} T \xi_{r}^{b} \xi_{h}^{c}+\frac{1}{2}\left(\Lambda_{b}^{2} \Lambda_{c}^{2} T-V_{a}\right) \xi_{h}^{b} \xi_{h}^{c}\right] \frac{d \xi_{r}^{a}}{d r}+\frac{1}{r^{2}}\left[S\left(\xi_{r}^{b}-\xi_{h}^{b}\right) \xi_{h}^{c}-F_{b} \xi_{r}^{b}\left(\xi_{r}^{c}-\xi_{h}^{c}\right)\right] \frac{d \xi_{h}^{a}}{d r}
$$

Symmetrizing this expression with respect to modes $b$ and $c$, employing equations (A4d)-(A4g), we get

$$
\oint d \Omega \operatorname{det}\left|\xi_{; j}^{i}\right|=-\frac{S}{3 r^{2}} \frac{d}{d r}\left(\xi_{h}^{a} \xi_{h}^{b} \xi_{h}^{c}\right)+\frac{\left(F_{a}+S\right)}{2 r^{2}} \frac{d}{d r}\left(\xi_{r}^{a} \xi_{h}^{b} \xi_{h}^{c}\right)-\frac{\Lambda_{a}^{2} T}{2 r^{2}} \frac{d}{d r}\left(\xi_{h}^{a} \xi_{r}^{b} \xi_{r}^{c}\right)+\frac{T}{3 r^{2}} \frac{d}{d r}\left(\xi_{r}^{a} \xi_{r}^{b} \xi_{r}^{c}\right)
$$

For gravity modes, $\left|\xi_{h}\right| \gg\left|\xi_{r}\right|$. Thus, the four terms in this expression decrease in size from left to right.

In a similar manner we arrive at

$$
\oint d \Omega(\nabla \cdot \xi)^{3}=T\left(\nabla \cdot \xi^{a}\right)\left(\nabla \cdot \xi^{b}\right)\left(\nabla \cdot \xi^{c}\right)
$$

and

$$
\begin{aligned}
\oint d \Omega(\nabla \cdot \xi) \xi_{; j}^{i} \xi_{; i}^{j}= & -\frac{F_{a}}{r}\left(\nabla \cdot \xi^{a}\right) \frac{d}{d r}\left(\xi_{h}^{b} \xi_{h}^{c}\right)+\frac{2 F_{a}}{r}\left(\nabla \cdot \xi^{a}\right) \xi_{r}^{b} \frac{d \xi_{h}^{c}}{d r}+T\left(\nabla \cdot \xi^{a}\right) \frac{d \xi_{r}^{b}}{d r} \frac{d \xi_{r}^{c}}{d r} \\
& +\frac{V_{a}}{r^{2}}\left(\nabla \cdot \xi^{a}\right) \xi_{h}^{b} \xi_{h}^{c}-\frac{2 T \Lambda_{c}^{2}}{r^{2}}\left(\nabla \cdot \xi^{a}\right) \xi_{r}^{b} \xi_{h}^{c}+\frac{2 T}{r^{2}}\left(\nabla \cdot \xi^{a}\right) \xi_{r}^{b} \xi_{r}^{c}
\end{aligned}
$$

The magnitudes of the terms in the above expression decrease from left to right except that the second, third, and fourth terms are of comparable size.

Having disposed of the angular dependences in $\kappa$, we turn to the radial integrations. The following relations prove helpful in this context:

$$
\begin{gathered}
\frac{d \xi_{r}}{d r}=(\nabla \cdot \xi)+\frac{\Lambda^{2}}{r} \xi_{h}-\frac{2}{r} \xi_{r}, \\
\frac{d}{d r}\left[\Gamma_{1} p(\nabla \cdot \xi)\right]=\frac{\Lambda^{2} \rho g}{r} \xi_{h}-\left(\omega^{2}+\frac{2 g}{r}-\frac{d g}{d r}\right) \rho \xi_{r}, \\
\Gamma_{1} p(\nabla \cdot \xi)=\rho g \xi_{r}-r \rho \omega^{2} \xi_{h} .
\end{gathered}
$$

The first equation is the definition of divergence, and the second and third are the radial and horizontal components of the equation of motion written in terms of the Lagrangian displacement.

Curvature terms arise because the directions of the basis vectors depend upon position. For instance,

$$
\xi_{; \theta}^{r}=\left(\xi_{r}-\xi_{h}\right) \frac{\partial Y_{\ell m}}{\partial \theta}
$$

where $\xi_{h}$ appears as a result of curvature in the coordinate system. The largest terms in equations (A6) and (A8) are curvature terms. However, their radial integrals cancel, leaving a much smaller net contribution. Direct numerical evaluation of equation (A1) leads to unreliable results, so it is important to carry out this cancellation analytically. Accordingly, we integrate each term by parts and apply equations (A4d) and (A9) to obtain

$$
\begin{aligned}
\int_{0}^{R} d r\left[\frac{S p}{3} \frac{d}{d r}\left(\xi_{h}^{a} \xi_{h}^{b} \xi_{h}^{c}\right)+\frac{F_{a} r \Gamma_{1} p}{2}\left(\nabla \cdot \xi^{a}\right) \frac{d}{d r}\left(\xi_{h}^{b} \xi_{h}^{c}\right)\right]= & -\int_{0}^{R} d r \frac{F_{a} \Gamma_{1} p}{2}\left(\nabla \cdot \xi^{a}\right) \xi_{h}^{b} \xi_{h}^{c} \\
& +\int_{0}^{R} d r \frac{F_{a} r \rho}{2}\left(\omega_{a}^{2}+\frac{2 g}{r}-\frac{d g}{d r}\right) \xi_{r}^{a} \xi_{h}^{b} \xi_{h}^{c}
\end{aligned}
$$

This step leads to

$$
\begin{aligned}
\kappa= & \int_{0}^{R} d r\left[-\frac{T^{2} \Gamma_{1}\left(\Gamma_{1}-2\right) p}{6}\left(\nabla \cdot \xi^{a}\right)\left(\nabla \cdot \xi^{b}\right)\left(\nabla \cdot \xi^{c}\right)-\frac{T r^{2} \Gamma_{1} p}{2}\left(\nabla \cdot \xi^{a}\right) \frac{d \xi_{r}^{b}}{d r} \frac{d \xi_{r}^{c}}{d r}\right. \\
& -F_{a} r \Gamma_{1} p\left(\nabla \cdot \xi^{a}\right) \xi_{r}^{b} \frac{d \xi_{h}^{c}}{d r}-\frac{\left(\Lambda_{b}^{2} \Lambda_{c}^{2} T-S\right) \Gamma_{1} p}{2}\left(\nabla \cdot \xi^{a}\right) \xi_{h}^{b} \xi_{h}^{c}-\frac{\left(S-F_{a}\right) \rho g}{2} \xi_{r}^{a} \xi_{h}^{b} \xi_{h}^{c}-\frac{F_{a} r \rho}{2} \frac{d g}{d r} \xi_{r}^{a} \xi_{h}^{b} \xi_{h}^{c} \\
& \left.+\Lambda_{a}^{2} T \rho g \xi_{h}^{a} \xi_{r}^{b} \xi_{r}^{c}+\Lambda_{c}^{2} T \Gamma_{1} p\left(\nabla \cdot \xi^{a}\right) \xi_{r}^{b} \xi_{h}^{c}-T p \frac{d \xi_{r}^{a}}{d r} \xi_{r}^{b} \xi_{r}^{c}-T \Gamma_{1} p\left(\nabla \cdot \xi^{a}\right) \xi_{r}^{b} \xi_{r}^{c}+\frac{\omega_{a}^{2} F_{a} r \rho}{2} \xi_{r}^{a} \xi_{h}^{b} \xi_{h}^{c}\right] .
\end{aligned}
$$


Next we systematically eliminate radial derivatives of the displacement vector from the expression for $\kappa$. We integrate by parts to dispose of $d \xi_{h} / d r$ and substitute for $d \xi_{r} / d r$ using equation (A9). With the aid of equations (A4d)-(A4f) and using equation (A11) to make the expression symmetric with respect to indices $b$ and $c$, we arrive at our final working expression for $\kappa$,

$$
\begin{aligned}
\kappa= & \int_{0}^{R} d r\left\{-\frac{\operatorname{Tr}^{2} \Gamma_{1}\left(\Gamma_{1}+1\right) p}{6}\left(\nabla \cdot \xi^{a}\right)\left(\nabla \cdot \xi^{b}\right)\left(\nabla \cdot \xi^{c}\right)+\frac{\omega_{a}^{2} G_{a} r \rho}{2} \xi_{h}^{a} \xi_{h}^{b} \xi_{h}^{c}\right. \\
& +\frac{F_{a} \rho}{2}\left(g-r \frac{d g}{d r}\right) \xi_{r}^{a} \xi_{h}^{b} \xi_{h}^{c}-\frac{\Lambda_{a}^{2} T r \Gamma_{1} p}{2} \xi_{h}^{a}\left(\nabla \cdot \xi^{b}\right)\left(\nabla \cdot \xi^{c}\right)-T\left(3 \Gamma_{1}+1\right) p\left(\nabla \cdot \xi^{a}\right) \xi_{r}^{b} \xi_{r}^{c} \\
& +2 \operatorname{Tr} \Gamma_{1} p \xi_{r}^{a}\left(\nabla \cdot \xi^{b}\right)\left(\nabla \cdot \xi^{c}\right)+\frac{2 T p}{r} \xi_{r}^{a} \xi_{r}^{b} \xi_{r}^{c}+\frac{1}{2}\left[\left(\omega_{a}^{2}-3 \omega_{b}^{2}-3 \omega_{c}^{2}\right) F_{a}-\left(2 \omega_{b}^{2} F_{b}+2 \omega_{c}^{2} F_{c}\right)\right] r \rho \xi_{r}^{a} \xi_{h}^{b} \xi_{h}^{c} \\
& \left.+\frac{1}{2}\left[\Lambda_{a}^{2} T\left(5 \rho g+\rho r \frac{d g}{d r}-\frac{2 p}{r}\right)-\rho\left(\omega_{b}^{2} F_{b}+\omega_{c}^{2} F_{c}\right) r\right] \xi_{h}^{a} \xi_{r}^{b} \xi_{r}^{c}\right\} .
\end{aligned}
$$

All permutations of the three modes are to be included when evaluating this expression. However, the largest contribution from each term comes when $b$ and $c$ are radially similar daughter modes. For high-order gravity modes, the first five terms are of comparable size and much larger than the remaining four. Numerical evaluation of $\kappa$ using equation (A15) does not suffer from the errors arising from large cancellations and numerical differentiation that plague attempts using equation (A1).

\section{A2. ORDER OF MAGNITUDE}

Here we estimate the maximum value that $\kappa$ can attain for parametric resonances involving untrapped parent and daughter modes. In so doing, we apply results derived in Papers I and II. These include (1) the scaling relations

$$
|\nabla \cdot \xi| \sim \frac{\Lambda^{2}}{R} \xi_{h} \sim \frac{\xi_{r}}{z_{\omega}} \approx k_{h} \xi_{h} \sim \frac{1}{\left(n \tau_{\omega} L\right)^{1 / 2}},
$$

in the evanescent region $z<z_{\omega}$, and

$$
|\nabla \cdot \xi| \sim\left(\frac{z_{\omega}}{z}\right)^{1 / 2} \frac{\Lambda^{2}}{R} \xi_{h} \sim \frac{\xi_{r}}{z}
$$

in the propagating cavity $z>z_{\omega}$; and (2) the fact that regions between consecutive radial nodes contribute equally to the following normalization integral:

$$
\frac{\omega^{2}}{2} \int_{0}^{R} d r r^{2} \rho\left(\Lambda^{2} \xi_{h}^{2}+\xi_{r}^{2}\right)=1
$$

As $\xi_{r}^{2} \ll \xi_{h}^{2}$ for $g$-modes, we have

$$
\omega^{2} R^{2} \int_{0}^{z} d z \rho \Lambda^{2} \xi_{h}^{2} \sim \frac{n^{\prime}}{n} \sim \frac{1}{n} \int_{0}^{z} d z k_{z} \sim \frac{1}{n}\left(\frac{z}{z_{\omega}}\right)^{1 / 2}
$$

Here $n^{\prime}$ is the number of radial nodes above depth $z$ and $n$ is the total number in the mode.

Equation (A15) yields maximal values for $\kappa$ when mode $a$ is taken to be the parent mode (subscript $p$ ) and modes $b$ and $c$ to be two radially similar daughter modes (subscripts $d_{1}$ and $d_{2}$ ). Most of the contribution to the radial integral comes from the region above $z_{\omega_{p}}$ : for parametric resonance, $z_{\omega_{p}}$ is much greater than $z_{\omega_{d_{1}}} \approx z_{\omega_{d_{2}}}$; the decay and rapid oscillation of the parent mode's eigenfunction render insignificant the contribution from greater depths. Thus, we can take the integrals in equation (A15) to run from $z=0$ to $z=z_{\omega_{p}}$ and pull out the parent mode eigenfunctions since they are approximately constant for $z<z_{\omega_{p}}$. This procedure reduces each of the leading terms in $\kappa$ and thus their sum to

$$
|\kappa|_{\max } \sim\left(\frac{z_{\omega_{p}}}{z_{\omega_{d}}}\right)^{1 / 2} \frac{1}{n_{d}\left(n_{p} \tau_{\omega_{p}} L\right)^{1 / 2}} .
$$

But $z_{\omega_{p}} / z_{\omega_{d}} \sim \Lambda_{d}^{2} / \Lambda_{p}^{2} \sim n_{d}^{2} / n_{p}^{2}$, which leads to

$$
|\kappa|_{\max } \sim \frac{1}{\left(n_{p}^{3} \tau_{\omega_{p}} L\right)^{1 / 2}} .
$$

The above equation establishes that $|\kappa|_{\max }$ rises steeply with increasing radial order of the parent mode and is independent of the radial orders and spherical degrees of the daughter modes. Our expression for $|\kappa|_{\max }$ applies to untrapped modes on both the high- and low-frequency branches of the $g$-mode dispersion relation (Paper I).

Trapping of either the parent or the daughter modes affects $|\kappa|_{\max }$. As an example, consider essentially complete trapping in the surface hydrogen layer of the parent mode and negligible trapping of its daughter modes. This requires $z_{\omega_{p}} \leq z_{\mathrm{H}}$, where 

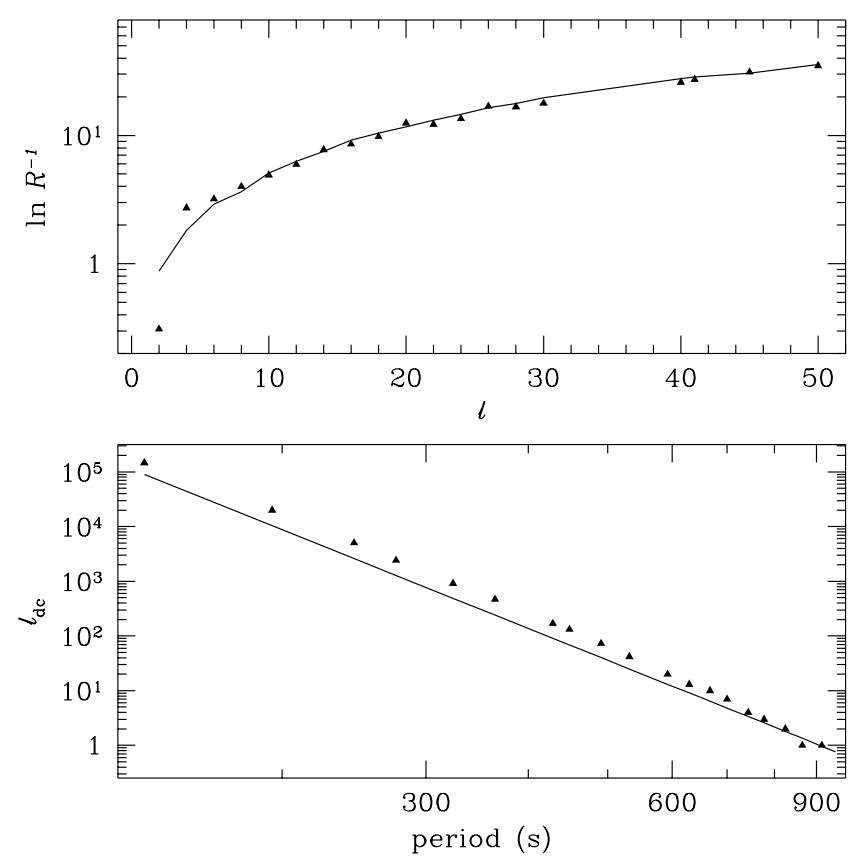

FIG. 10.-Strongly nonadiabatic modes in the hottest model considered in Fig. 5. The top panel exhibits the dependence of the reflection coefficient, $\mathscr{R}$, on $\ell$ for modes with periods close to $550 \mathrm{~s}$. The numerical result for the reflection coefficient is obtained from numerical computations of $\gamma$ and is depicted by filled triangles. The underlying solid line is the theoretical estimate (eq. [B7]). Computing $\gamma$ requires care since $\mathscr{R} \approx 10^{-16}$ for $\ell=50$. This is of the order of the machine accuracy for double precision numbers. The bottom panel shows the angular degree at which decoupling occurs, $\ell_{\mathrm{dc}}$, as a function of period for $\ell=1$ parent modes. Filled triangles denote numerical values and the solid line the analytical scaling relation given by eq. (B8). We find that $\ell_{\mathrm{dc}}$ is almost independent of the star's effective temperature.

$z_{\mathbf{H}}$ is the depth at the base of the hydrogen layer. Under these conditions, the derivation of the analytic estimate for $|\kappa|_{\max }$ remains valid through equation (A20) provided we replace $n_{p}$ by $n_{p}^{\prime}$, the number of parent mode nodes above $z_{\mathrm{H}}$. The step leading to equation (A20) now yields

$$
|\kappa|_{\max } \sim \frac{1}{n_{p}\left(n_{p}^{\prime} \tau_{\omega_{p}} L\right)^{1 / 2}} .
$$

For later reference we note that the parent mode normalization factor (see eq. [16]) carries an $n_{p}^{\prime}$ in place of $n_{p}$. Thus, the ratio of $\kappa_{\max }^{2}$ to the parent mode normalization factor remains equal to $n_{p}^{-1}$.

\section{APPENDIX B}

\section{STRONGLY NONADIABATIC DAUGHTER MODES}

Strong nonadiabaticity occurs wherever

$$
\frac{\omega \tau_{\mathrm{th}}}{\left(k_{z} z\right)^{2}} \lesssim 1
$$

We refer to the depth above which this inequality applies as $z_{\text {na }}$. To derive a simple analytic scaling relation for $z_{\text {na }}$, we make use of the approximations $k_{z} \sim\left(z z_{\omega}\right)^{-1 / 2}$ and $\tau_{\mathrm{th}} / \tau_{b} \sim\left(z / z_{b}\right)^{q+2}$. Here $z_{b}$ and $\tau_{b}$ are the depth and thermal relaxation time at the bottom of the convection zone, and $\rho \propto z^{q}$ with $q \approx 3.5$ provides a fit to the density structure in the upper portion of the radiative interior. It then follows that

$$
\frac{z_{\text {na }}}{z_{b}} \sim\left(\frac{1}{\omega \tau_{b}} \frac{z_{b}}{z_{\omega}}\right)^{1 /(q+1)}
$$

Moreover,

$$
\left(k_{z} z\right)_{\mathrm{na}} \sim\left[\frac{1}{\left(\omega \tau_{b}\right)}\left(\frac{z_{b}}{z_{\omega}}\right)^{q+2}\right]^{1 /[2(q+1)]} .
$$

By reducing the effective buoyancy, strong nonadiabaticity lowers the effective lid of a $g$-mode's cavity to $z_{\text {na }}$. Consequences of this fact are explored in the following subsections. 


\section{B1. DAMPING RATES OF STRONGLY NONADIABATIC MODES}

As shown in Paper II, the energy dissipation rate for a strongly nonadiabatic mode may be written as

$$
\gamma \approx \frac{\omega}{\pi n} \ln \mathscr{R}^{-1}
$$

where $\mathscr{R}$ denotes the coefficient of amplitude reflection at the top of the mode's cavity.

To derive an approximate relation for $\mathscr{R}$, we note that the real and imaginary parts of $k_{z}, k_{z r}$ and $k_{z i}$, satisfy

$$
\frac{\left|k_{z i}\right|}{\left|k_{z r}\right|} \sim \frac{\left(k_{z r} z\right)^{2}}{\omega \tau_{\mathrm{th}}}
$$

provided $\left|k_{z i}\right| /\left|k_{z r}\right| \lesssim 1$. Thus,

$$
\ln \mathscr{R}^{-1} \sim \int_{z_{\mathrm{na}}}^{\infty} d z\left|k_{z i}\right| .
$$

Evaluating this integral with the aid of equations (B2) and (B3), we obtain

$$
\ln \mathscr{R}^{-1} \sim\left[\frac{1}{\left(\omega \tau_{b}\right)}\left(\frac{z_{b}}{z_{\omega}}\right)^{q+2}\right]^{1 /[2(q+1)]} \propto \frac{\ell^{(q+2) /(q+1)}}{\omega^{(2 q+5) /(2 q+2)}} .
$$

Numerical results for $\ln \mathscr{R}^{-1}$ plotted in the upper panel of Figure 10 confirm this relation.

Because the maximum value of $\kappa$ for parametric resonance is independent of the spherical degrees of the daughter modes, the dependence of $\gamma$ on $\ell$ at fixed $\omega$ is of great significance. Equations (B4) and (B7), together with the relation $n \propto \ell$ at fixed $\omega$, establish that $\gamma \propto \ell^{1 /(q+1)}$. Since $\gamma$ increases with $\ell$ at fixed $\omega$, the most important daughter pairs are those with the smallest $\ell$ values subject to the constraint $\gamma_{d}>\delta \omega$.

\section{B2. REDUCTION OF THE COUPLING COEFFICIENT BY STRONG NONADIABATICITY}

The maximum adiabatic coupling coefficient between a parent mode and a pair of daughter modes is $\kappa_{\max } \sim\left(n_{p}^{3} \tau_{\omega_{p}} L\right)^{-1 / 2}$ (eq. [12]). Its major contribution comes from the region above $z_{\omega_{p}}$. The factor $\left(n_{p} \tau_{\omega_{p}} L\right)^{-1 / 2}$ is the surface value of the normalized eigenfunction for $[\ell(\ell+1)]^{1 / 2} \xi_{h} / R$. The extra factor $n_{p}^{-1^{\omega_{p}}}$ is the fraction of each daughter mode's nodes that lie above $z_{\omega_{p}}$. The coupling coefficient is reduced compared to equation (12) for daughter modes that are strongly nonadiabatic in the region above $z_{\omega_{p}}$.

Equation (B1) indicates that nonadiabaticity increases with increasing $\ell$ at fixed $z$ and $\omega ; k_{z} \sim\left(z z_{\omega}\right)^{-1 / 2}$ and $z_{\omega} \sim$ $\omega^{2} R^{2} / g \ell(\ell+1)$, so $k_{z} \propto \ell$. We define the angular degree of decoupling for a given parent mode, $\ell_{\mathrm{dc}}$, as the smallest spherical degree at which its daughter modes are strongly nonadiabatic all the way down to the top of the parent mode's cavity, that is, $z_{\mathrm{na}_{d}} \approx z_{\omega_{p}}$ at $\ell_{\mathrm{dc}}$. Using equation (B2), we find

$$
\ell_{\mathrm{dc}} \sim\left\{\left(\frac{\omega^{2} R^{2}}{g z_{b}}\right)^{q+2} \frac{\omega \tau_{b}}{\left[\ell_{p}\left(\ell_{p}+1\right)\right]^{q+1}}\right\}^{1 / 2} .
$$

Numerical results displayed in the lower panel of Figure 10 are well represented by this scaling relation. We find that $\ell_{\mathrm{dc}}$ is relatively independent of stellar effective temperature but decays steeply with mode period. By $n_{p}=20$, we find $\ell_{\mathrm{dc}} \leq 1$.

It is plausible that at $\ell_{d}=\ell_{\mathrm{dc}}, \kappa_{\max }$ is reduced by a factor $n_{p} / n_{d}$ below its adiabatic value (eq. [12]) because the effective lids of the daughter modes' cavities are lowered to $z_{\omega_{p}}$. Since $n_{p} / n_{d} \approx \ell_{p} / 2 \ell_{d}$, this is a large reduction for $\ell_{\mathrm{dc}} \gg \ell_{p}$. An even more severe reduction of $\kappa_{\max }$ is expected for $\ell_{d}>\ell_{\mathrm{dc}}$.

\section{B3. PARAMETRIC INSTABILITY FOR TRAVELING WAVES}

In the limit of strong nonadiabaticity, daughter modes are more appropriately described as traveling waves than as standing waves. Thus, it behooves us to investigate the parametric instability of traveling waves. Here we demonstrate that the instability criterion for traveling waves is equivalent to that for standing waves (eq. [4]).

Nonlinear interactions between parent and daughter modes are localized within an interaction region above $z_{\omega_{p}}$. Let us assume that $z_{\mathrm{na}_{d}} \ll z_{\omega_{p}}$. Then, in most of the interaction region daughter wave packets may be represented as linear superpositions of adiabatic modes. Propagating at their group velocity, the daughter wave packets pass through the interaction region in a time interval

$$
\Delta T=\int_{0}^{z_{\omega_{p}}} \frac{d z}{v_{g z}} \sim \frac{1}{n_{p}} \frac{\pi n_{d}}{\omega_{d}} .
$$

Three significant relations involving $n_{p}$ are worth noting: (1) $n_{p}^{-1}$ is the fraction of each daughter mode's nodes that lie above $z_{\omega_{p}}$, so $2 \Delta T$ is a fraction $n_{p}^{-1}$ of the time each daughter wave packet takes to make a round trip across its cavity; (2) approximately $n_{p}$ daughter modes reside within the frequency interval $\pi / \Delta T$, and their relative phases change by less than $\pi$ as each daughter wave packet crosses the interaction region; and (3) maximal $\kappa$ occurs inside an interval of width $\left|n_{d_{1}}-n_{d_{2}}\right| \lesssim$ $n_{p}$.

Nonlinear interactions between parent and daughter waves within the interaction region are described by equations (1)-(3) with two modifications of the equations governing the time evolution of the daughter modes. The linear damping term is 
negligible for $z \gg z_{\text {na }}$, and the nonlinear term must be multiplied by a factor $n_{p}$. The latter accounts for the number of modes that couple coherently to each daughter mode during the interaction time $\Delta T$. During two passes through the interaction region, the amplitudes of the daughter wave packets grow by a factor $e^{\mathscr{G}}$, where the gain, $\mathscr{G}$, is given by

$$
\mathscr{G}=\frac{2 \Delta T}{\left|A_{d}\right|} \frac{d\left|A_{d}\right|}{d t} \approx 3 \sqrt{2} n_{d}|\kappa|\left|A_{p}\right|
$$

For parametric instability to occur,

$$
\mathscr{G}>\ln \mathscr{R}^{-1} .
$$

Combining the relation between $\mathscr{R}$ and $\gamma$ given by equation (B4) with equation (B11), the threshold condition for parametric instability of traveling waves becomes

$$
\left|A_{p}\right|>\frac{\gamma_{d}}{3 \sqrt{2} \omega_{d}|\kappa|} .
$$

The above condition is equivalent to equation (4) in the limit that $\gamma_{d} \gg \delta \omega$. Thus, the threshold condition for parametric instability of traveling waves reduces to a limiting case of the threshold condition for parametric instability of standing waves.

Bradley, P. A. 1996, ApJ, 468, 350

Brickhill, A. J. 1990, MNRAS, 246, 510 .1991, MNRAS, 251, 673

Clemens, J. C. 1993, Baltic Astron., 2, 407 1995, Baltic Astron., 4, 142

Dziembowski, W. 1982, Acta Astron., 32, 147

Dziembowski, W., \& Krolikowska, M. 1985, Acta Astron., 35, 5

Goldreich, P., \& Wu, Y. 1998, ApJ, 511, 904 (Paper I) 1999, ApJ, 523, 805 (Paper III)

Jensen, B. L., Sumer, B. M., \& Fredsoe, J. 1989, J. Fluid Mech., 206, 265

Kepler, S. O., et al. 2000, ApJ, 534, L185
REFERENCES

Kleinman, S. J., et al. 1998, ApJ, 495, 424

Kumar, P., \& Goldreich, P. 1989, ApJ, 342, 558

Kumar, P., \& Goodman, J. 1996, ApJ, 466, 946

Landau, L. D., \& Lifshitz, E. M. 1976, in Mechanics (3d ed.; New York: Pergamon Press), 80

Newcomb, W. A. 1962, Nuclear Fusion: Supplement Part 2, Vienna: International Atomic Energy Agency, 451

Vandakurov, V. V. 1979, AZh, 56, 749

Wersinger, J. M., Finn, J. M., \& Ott, E. 1980, Phys. Fluids, 23, 1142

Winget, D. E., et al. 1994, ApJ, 430, 839

Wu, Y., \& Goldreich, P. 1999, ApJ, 519, 783 (Paper II) 